\title{
STABILITY ESTIMATES FOR AN INVERSE PROBLEM FOR THE MAGNETIC SCHRÖDINGER OPERATOR
}

\author{
PEDRO CARO AND VALTER POHJOLA
}

\begin{abstract}
In this paper we prove stable determination of an inverse boundary value problem associated to a magnetic Schrödinger operator assuming that the magnetic and electric potentials are essentially bounded and the magnetic potentials admit a Hölder-type modulus of continuity in the sense of $L^{2}$.
\end{abstract}

\section{Contents}

1. Introduction and main results

2. From the boundary to the interior

3. Complex geometric optics solutions

4. Stability estimates for the magnetic fields

5. Stability estimates for the electric potentials

6. Estimating the co-exact part of the magnetic potential

References

\section{INTRODUCTION AND MAIN RESULtS}

Let $U$ be a bounded non-empty open subset of $\mathbb{R}^{n}$ (from now on a domain) with $n \geq 3$. Given a magnetic potential $A$ and an electric potential $q$, in the Lebesgue spaces $L^{\infty}\left(U ; \mathbb{C}^{n}\right)$ and $L^{\infty}(U)$ respectively, we consider the magnetic Schrödinger operator formally given by

$$
L_{A, q} u=-(\nabla+i A) \cdot(\nabla+i A) u+q u .
$$

This corresponds to the operator $L_{A, q}^{U}: H^{1}(U) \longrightarrow H^{-1}(U)$ given by

$$
\left\langle L_{A, q}^{U} u, v\right\rangle=\int_{U} \nabla u \cdot \nabla v+i A \cdot(u \nabla v-v \nabla u)+(A \cdot A+q) u v d x
$$

for any $u \in H^{1}(U)$ and $v \in H_{0}^{1}(U)$. Here $H^{1}(U)$ denotes the first order Sobolev space based in the Lebesgue space $L^{2}(U)$. The space $H_{0}^{1}(U)$ denotes the closure in $H^{1}(U)$, of the compactly supported smooth functions in $U$ and $H^{-1}(U)$ denotes its dual. For convenience, $A \cdot A$ will be denoted by $A^{2}$ and we will write $L_{A, q}$ instead of $L_{A, q}^{U}$ whenever the

Date: January 14, 2013.

Key words and phrases. Inverse problems; Magnetic Scrödinger; stability.

MSC: 35R30. 
domain associated to this definition is clear. Note also that $L_{A, q}$ is linear and bounded.

Next we describe the boundary data of a $H^{1}(U)$ solution $u$ to the magnetic Schrödinger equation

$$
L_{A, q} u=0
$$

and then we define the Cauchy data set associated to this equation. It is well known that the trace space of $H^{1}(U)$, denoted here by $T H^{1}(U)$, is described by the quotient $H^{1}(U) / H_{0}^{1}(U)$. The space $T H^{1}(U)$ endowed with the quotient norm, denoted by $\|\cdot\|_{T H^{1}(U)}$, is a Banach space. The trace map $T_{U}: H^{1}(U) \longrightarrow T H^{1}(U)$ is defined by $T_{U} u=[u]$ for any $u \in$ $H^{1}(U)$, where $[u]$ denotes the equivalence class of $u$. For convenience, we will write $T$ instead of $T_{U}$ whenever the associated domain is clear.

The normal component of the magnetic gradient on the boundary is, in a regular enough setting, given by $\left.\left(\partial_{\nu}+i \nu \cdot A\right) u\right|_{\partial U}$ (where $\nu$ denotes the outward pointing unit normal vector on the boundary of $U$ denoted by $\partial U)$. In our case we define this following [15], as the bounded linear map $N_{A, q}^{U}: H^{1}(U) \longrightarrow T H^{1}(U)^{*}$ given by

$$
\left\langle N_{A, q}^{U} u, g\right\rangle=\int_{U} \nabla u \cdot \nabla v+i A \cdot(u \nabla v-v \nabla u)+\left(A^{2}+q\right) u v d x
$$

for any $u \in H^{1}(U)$ such that $L_{A, q} u=0$ and any $g \in T H^{1}(U)$ such that $g=T v$. The space $\left(T H^{1}(U)^{*},\|\cdot\|_{T H^{1}(U)^{*}}\right)$ denotes the dual space of $\left(T H^{1}(U),\|\cdot\|_{T H^{1}(U)}\right)$. Again, we will write $N_{A, q}$ instead of $N_{A, q}^{U}$ whenever the domain is clear. Finally, the Cauchy data set of $H^{1}(U)$ solutions to the magnetic Schrödinger equation is defined as

$$
C_{A, q}=\left\{\left(T u, N_{A, q} u\right): u \in H^{1}(U), L_{A, q} u=0\right\} .
$$

From now on, $C_{A, q}$ will be referred as the Cauchy data set associated to the operator $L_{A, q}$. Note that $C_{A, q}$ encodes the information of the solutions on the boundary of $U$, hence it is usually called boundary measurements.

The inverse boundary value problem (IBVP for short) considered in this paper consists in recovering the magnetic and electric potentials from the knowledge of their associated Cauchy data set. Related to this problem, some other natural questions arise as uniqueness and stability.

The first question can be stated as follows: Given two magnetic potentials $A_{1}, A_{2} \in L^{\infty}\left(U ; \mathbb{C}^{n}\right)$ and two electric potentials $q_{1}, q_{2} \in L^{\infty}(U)$, does $C_{A_{1}, q_{1}}=C_{A_{2}, q_{2}}$ imply $A_{1}=A_{2}$ and $q_{1}=q_{2}$ ? The answer to this question is negative because of the following obstruction. For every $\varphi$ in the space $1 \operatorname{Lip}(1, \bar{U})$ with $\varphi(x)=0$ for all $x \in \partial U$, one has $C_{A+\nabla \varphi, q}=C_{A, q}$ (see [15] for details). Thus, from the boundary

\footnotetext{
${ }^{1}$ The space $\operatorname{Lip}(1, \bar{U})$ is the space of Lipschitz continuous functions, see 20] for the precise definition.
} 
measurements one can not distinguish between $A$ and $A+\nabla \varphi$. This problem does however not effect the magnetic field $d A$, which is interpreted as follows. Recall that any vector field $A \in L^{\infty}\left(U ; \mathbb{C}^{n}\right)$ with components $A_{j}$ can be identified with the 1 -form

$$
\sum_{j=1}^{n} A_{j} d x_{j}
$$

still denoted by $A$. The magnetic field induced by the potential $A$ is now given by

$$
d A=\sum_{1 \leq j<k \leq n}\left(\partial_{x_{j}} A_{k}-\partial_{x_{k}} A_{j}\right) d_{x_{j}} \wedge d_{x_{k}} .
$$

Due to the lack of smoothness of $A$, this definition has to be understood in the sense of currents (i.e. differential forms in the sense of distributions). The magnetic potentials $A$ and $A+\nabla \varphi$ induce the same magnetic field, since $d(A+d \varphi)=d A$ (where $\left.d \varphi=\sum_{1}^{n} \partial_{x_{j}} \varphi d x_{j}\right)$. The non uniqueness described above does therefore not extend to the magnetic fields. Thus, the problem we will consider consists in recovering the magnetic field $d A$ and the electric potential $q$ from the Cauchy data set.

The question of stability essentially ask whether it is possible to provide a quantitative answer to the (qualitative) question of uniqueness. More precisely, if the proximity of the magnetic fields and electric potentials can be estimated by the proximity of their corresponding Cauchy data sets. In order to study the question of stability, one should have some notion of proximity between Cauchy data sets. Let $A_{1}, A_{2} \in L^{\infty}\left(U ; \mathbb{C}^{n}\right)$ be two magnetic potentials and let $q_{1}, q_{2} \in L^{\infty}(U)$ be two electric potentials. Consider $C_{A_{j}, q_{j}}$ the Cauchy data set associated to $L_{A_{j}, q_{j}}$ with $j \in\{1,2\}$. Given $\left(f_{j}, g_{j}\right) \in C_{A_{j}, q_{j}}$ with $j \in\{1,2\}$ set

$$
I\left(\left(f_{j}, g_{j}\right) ; C_{A_{k}, q_{k}}\right)=\inf _{\left(f_{k}, g_{k}\right) \in C_{A_{k}, q_{k}}}\left[\left\|f_{j}-f_{k}\right\|_{T H^{1}(U)}+\left\|g_{j}-g_{k}\right\|_{T H^{1}(U)^{*}}\right],
$$

with $k \in\{1,2\}$. We define the pseudo-metric distance between $C_{A_{1}, q_{1}}$ and $C_{A_{2}, q_{2}}$ as

$$
\operatorname{dist}\left(C_{A_{1}, q_{1}}, C_{A_{2}, q_{2}}\right)=\max _{j, k \in\{1,2\}} \sup _{\substack{\left(f_{j}, g_{j}\right) \in C_{A_{j}, q_{j}} \\\left\|f_{j}\right\|_{T H^{1}(U)}=1}} I\left(\left(f_{j}, g_{j}\right) ; C_{A_{k}, q_{k}}\right) .
$$

This notion of proximity was introduced in [4] and it has been successfully used to study the stability of certain IBVP on frameworks where the forward problem is ill-posed (see [5] and [14]).

The uniqueness and the stability of this IBVP have been studied by several authors under various regularity assumptions on the magnetic and electric potentials. In [21, a local uniqueness result was established for magnetic potentials in $W^{2, \infty}$ and $L^{\infty}$ electric potentials -the local 
nature of the result is due to a smallness condition imposed to the magnetic potential. In [16], the smallness condition was removed for smooth magnetic and electric potentials, and for compactly supported $C^{2}$ magnetic potentials and $L^{\infty}$ electric potentials. The uniqueness results were subsequently extended to $C^{1}$ magnetic potentials in [23], to some less regular but small potentials in [17], and to Dini continuous magnetic potentials in [18]. The best result by now is [15], by Krupchyk and Uhlmann, where they proved uniqueness assuming the magnetic and electric potentials to be essentially bounded. Furthermore, they do not require regularity assumptions for the boundary of the domain. Uniqueness for the closely related inverse scattering problem with a magnetic potential has been studied by Eskin and Ralston in [9].

The question of stability has been studied in [25] by Tzou. There, a log-type stability estimate is established for the IBVP studied in this paper, assuming that the boundary of the domain is smooth, the magnetic potentials are in $W^{2, \infty}$ with equal values on the boundary and the electric potentials are in $L^{\infty}$.

The questions of uniqueness, stability and reconstruction for nonsmooth frameworks have been recently studied for several IBVP as the Calderón problem (see [2], [8] and [11] for dimension $n=2$ and [3], [13], [6] and [12] for $n \geq 3$ ) and for an IBVP associated to the time-harmonic Maxwell equations (see [7]).

In this paper, we consider the question of stability associated to the previously described IBVP. We improve considerably the stability result by Tzou providing a quantitative version of the result proved by Krupchyk and Uhlmann. In order to state precisely our result, we need to introduce some notation.

Given a domain $\Omega$ in $\mathbb{R}^{n}$ and two constants $M \in[1,+\infty)$ and $\varepsilon \in$ $(0,1)$, we define the class of admissible magnetic potentials, denoted by $\mathscr{A}(\Omega, M, \varepsilon, r)$ with $r \in[1,+\infty)$ or $r=\infty$, as the class of $A \in$ $L^{\infty}\left(\Omega ; \mathbb{C}^{n}\right)$ such that its extension by zero out of $\Omega$, still denoted by $A$, satisfies the a priori bound

$$
\|A\|_{L^{\infty}\left(\mathbb{R}^{n} ; \mathbb{C}^{n}\right)}+|A|_{B_{\varepsilon}^{2, r}} \leq M
$$

Here

$$
|A|_{B_{\varepsilon}^{2, r}}^{2}=\sum_{j=1}^{n}\left(\int_{\mathbb{R}^{n}} \frac{\left\|A_{j}(\cdot+y)-A_{j}\right\|_{L^{2}\left(\mathbb{R}^{n}\right)}^{r}}{|y|^{n+r \varepsilon}} d y\right)^{2 / r}
$$

for $r \in[1,+\infty)$ and

$$
|A|_{B_{\varepsilon}^{2, \infty}}^{2}=\sum_{j=1}^{n} \sup _{y \in \mathbb{R}^{n} \backslash\{0\}} \frac{\left\|A_{j}(\cdot+y)-A_{j}\right\|_{L^{2}\left(\mathbb{R}^{n}\right)}^{2}}{|y|^{2 \varepsilon}}
$$


with $A_{j}$ denoting $j$-th component of $A$. Note that if $\partial \Omega$ can be locally described by the graph of a Lipschitz function and

$$
\|A\|_{L^{\infty}\left(\Omega ; \mathbb{C}^{n}\right)}+|A|_{B_{\varepsilon}^{2, r}(\Omega)} \leq M
$$

then the extension by zero of $A$ out of $\Omega$ will satisfies (see [24]) an a priori bound depending on $M$ as well as $n$ and $\Omega$. The same should happen for more general boundaries. This has been studied in [1] for the case of Sobolev spaces $W^{s, p}(\Omega)$.

For $\Omega$ and $M$ as above, we also define the class of admissible electric potentials $\mathscr{Q}(\Omega, M)$ as the class of $q \in L^{\infty}(\Omega)$ such that

$$
\|q\|_{L^{\infty}(\Omega)}<M \text {. }
$$

The extension by zero out of $\Omega$ of $q \in \mathscr{Q}(\Omega, M)$ will be also denoted by $q$.

Theorem 1.1. Let $\Omega$ be a domain in $\mathbb{R}^{n}$ and consider two constants $M \in[1,+\infty)$ and $\varepsilon \in(0,1)$. There exists a constant $c_{0} \in(0,1)$, depending on $M$ and $\Omega$ such that if $A_{1}, A_{2} \in \mathscr{A}(\Omega, M, \varepsilon, r)$ with $r \in$ $[1,+\infty)$ or $r=\infty, q_{1}, q_{2} \in \mathscr{Q}(\Omega, M), C_{j}$ denotes the Cauchy data set associated to $A_{j}, q_{j}$ and $\left|\log \operatorname{dist}\left(C_{1}, C_{2}\right)\right|^{-1}<c_{0}$, then

$$
\left\|d A_{1}-d A_{2}\right\|_{H^{-1} \mathbf{\Omega}^{2}\left(\mathbb{R}^{n}\right)} \lesssim\left|\log \operatorname{dist}\left(C_{1}, C_{2}\right)\right|^{-c \varepsilon^{2} / n}
$$

with $c \in(0,1)$ universal. Moreover, if $\delta \in(1-\varepsilon, 1)$ then

$$
\left\|d A_{1}-d A_{2}\right\|_{B_{-\delta}^{2, r} \Omega^{2}\left(\mathbb{R}^{n}\right)} \lesssim\left|\log \operatorname{dist}\left(C_{1}, C_{2}\right)\right|^{-c \varepsilon(\delta-1+\varepsilon) / n} .
$$

The implicit constant in these estimates depend on $M$ and $\varepsilon$ as well as on $n$ and $\Omega$. The implicit constant on the second one also depends on $\delta$.

The symbol $\lesssim$ holds for $\leq$ modulo a multiplicative constant. This constant is called here implicit constant. On the other hand, if $X(G)$ with $G$ a non-empty open subset of $\mathbb{R}^{n}$ denotes a function space, then $X \Omega^{k}(G)$ denotes the corresponding space for differential forms of degree $k$. In particular, the definitions of $H^{-1} \Omega^{2}\left(\mathbb{R}^{n}\right)$ and $B_{-\delta}^{2, r} \Omega^{2}\left(\mathbb{R}^{n}\right)$ can be found right before Proposition 4.2 and Proposition 4.3 in Section 4 . respectively.

Theorem 1.2. Consider $\lambda \in(0,1]$ and $\theta \in(0,2 / n)$. Under the same assumptions as in Theorem 1.1 we have that there exists a constant $c_{0} \in$ $(0,1)$ depending on $M, \Omega, n, \theta$ and $\varepsilon$ such that if $\left|\log \operatorname{dist}\left(C_{1}, C_{2}\right)\right|^{-1}<$ $c_{0}$, then

$$
\left\|q_{1}-q_{2}\right\|_{H^{-\lambda}\left(\mathbb{R}^{n}\right)} \lesssim\left|\log \operatorname{dist}\left(C_{1}, C_{2}\right)\right|^{-c \theta \varepsilon^{3} \lambda / n^{2}}
$$

with $c \in(0,1)$ universal. Moreover, if $q_{j} \in B_{\varepsilon}^{2, r}\left(\mathbb{R}^{n}\right)$ and satisfies the a priori bound $\left\|q_{j}\right\|_{B_{\varepsilon}^{2, r}\left(\mathbb{R}^{n}\right)}<M$ then

$$
\left\|q_{1}-q_{2}\right\|_{B_{0}^{2, r}\left(\mathbb{R}^{n}\right)} \lesssim\left|\log \operatorname{dist}\left(C_{1}, C_{2}\right)\right|^{-c \theta \varepsilon^{4} / n^{2}} .
$$


The implicit constant in these estimates depend on $M, \varepsilon$ and $\theta$ as well as on $n$ and $\Omega$. The implicit constant on the first one also depends on $\lambda$.

The definition of the spaces $H^{-\lambda}\left(\mathbb{R}^{n}\right)$ and $B_{0}^{2, r}\left(\mathbb{R}^{n}\right)$ can be found right before Proposition 5.4 and Proposition 5.5 in Section 5, respectively. On the other hand, $f \in B_{\varepsilon}^{2, r}\left(\mathbb{R}^{n}\right)$ if $f \in L^{2}\left(\mathbb{R}^{n}\right)$ and $|f|_{B_{\varepsilon}^{2, r}}<\infty$. Note that $|\cdot|_{B_{\varepsilon}^{2, r}}$ has been defined for vector fields, the definition for functions is similar.

Theorem 1.1 is consequence of Proposition 4.2 and Proposition 4.3 . The stability estimates there are stated for norms with negative index since $d A_{j}$ has to be understood in a weak sense. On the other hand, Theorem 1.2 is consequence of Proposition 5.4 and Proposition 5.5. The first stability estimate is stated for the norm of $H^{-\lambda}\left(\mathbb{R}^{n}\right)$ but, assuming an a priori upper bound on the norm $H^{\varepsilon}\left(\mathbb{R}^{n}\right)$ of the potentials, one could deduce a stability estimate controlling the $L^{2}\left(\mathbb{R}^{n}\right)$-norm of the potentials. We should not expect to prove a stability estimate controlling the $L^{\infty}\left(\mathbb{R}^{n}\right)$-norm since the potentials are not continuous.

Regarding the second parts of the estimates in Theorem 1.1 and Theorem 1.2, it is worth to point out that whenever $r=2$ the norm of the spaces $B_{-\delta}^{2, r} \Omega^{2}\left(\mathbb{R}^{n}\right)$ and $H^{-\delta} \Omega^{2}\left(\mathbb{R}^{n}\right)$ and the spaces $B_{0}^{2, r}\left(\mathbb{R}^{n}\right)$ and $L^{2}\left(\mathbb{R}^{n}\right)$ are equivalent respectively. Thus, the second estimates in the theorems generalize the ones we would get by interpolation between the first estimates in the theorems and the corresponding a priori bounds.

Let us now explain the main difficulties and ideas in the proofs of Theorem 1.1 and Theorem 1.2. We start by recalling the qualitative argument due to Krupchyk and Uhlmann. Their starting point is the following integral identity

$$
\int_{\Omega} i\left(A_{1}-A_{2}\right) \cdot\left(u_{1} \nabla \overline{u_{2}}-\overline{u_{2}} \nabla u_{1}\right)+\left(A_{1}^{2}-A_{2}^{2}+q_{1}-q_{2}\right) u_{1} \overline{u_{2}} d x=0,
$$

which holds for $u_{1}$ and $u_{2}$ solving $L_{A_{1}, q_{1}} u_{1}=0$ and $L_{\overline{A_{2}}, \overline{q_{2}}} u_{2}=0$ respectively, whenever $C_{A_{1}, q_{1}}=C_{A_{2}, q_{2}}$. They then proceed by constructing so called complex geometric optics solutions (CGOs for short) that are to be used with the integral identity. The CGOs are solutions of the form

$$
u=e^{\zeta \cdot x / h}(a+r(h))
$$

where $\zeta$ is a complex vector, $h$ is a small parameter, $a$ is a sort of complex amplitude and $r(h)$ is a correction term that vanishes when $h$ goes to zero. With the CGOs and the integral identity at hand, they deduce that $d A_{1}=d A_{2}$. The next step for them was to prove that $q_{1}=q_{2}$. Using the fact that $d A_{1}=d A_{2}$ is unfortunately not by itself enough to remove the $A_{1}$ and $A_{2}$ terms from the integral identity and isolate the term containing $q_{1}-q_{2}$. They solved this problem by using the Poincaré lemma for currents to conclude that $A_{1}=A_{2}+\nabla \varphi$, since $d A_{1}=d A_{2}$. This allowed them to consider the pair of potentials 
$\left(A_{1}, q_{1}\right)$ and $\left(A_{1}-\nabla \varphi, q_{2}\right)$, instead of the original ones. Then, they exploited the gauge invariance of the Cauchy data sets in a ball $B$ containing $\bar{\Omega}$, by picking a $\varphi$ that vanishes on the boundary $\partial B$, to conclude that $C_{A_{1}, q_{1}}=C_{A_{1}-\nabla \varphi, q_{2}}$, and hence that $C_{A_{1}, q_{1}}=C_{A_{1}, q_{2}}$. Thus they could assume that $A_{1}=A_{2}$ in the above integral identity and they could isolate the term containing $q_{1}-q_{2}$ to prove that $q_{1}=q_{2}$.

Krupchyk and Uhlmann's construction of CGOs is based on the use of Carleman estimates, and its main feature is that they only need to make approximation of the magnetic potentials by smooth vector fields in the $L^{2}$ sense 2 Regarding a quantitative counterpart of Krupchyk and Uhlmann's approach, the first point will be to find an appropriate class of magnetic potentials for which the rate of approximation by smooth vector fields in the $L^{2}$ sense (with respect to $h$ ) is the same. To do this, we only need to prescribe an $L^{2}$ modulus of continuity and define the class as all the magnetic potentials admitting this modulus of continuity. However, in order to obtain the optimal stability for this IBVP, namely log type, we need to assume that this modulus of continuity is of Hölder type, say of order $\varepsilon$. This suggests examining magnetic potentials in the Besov spaces $B_{\varepsilon}^{2, r}$. With this choice one can then relatively straight forwardly prove stability for the magnetic fields using the following integral estimate

$$
\begin{gathered}
\left|\int_{\Omega} i\left(A_{1}-A_{2}\right) \cdot\left(u_{1} \nabla \overline{u_{2}}-\overline{u_{2}} \nabla u_{1}\right)+\left(A_{1}^{2}-A_{2}^{2}+q_{1}-q_{2}\right) u_{1} \overline{u_{2}} d x\right| \\
\lesssim \operatorname{dist}\left(C_{1}, C_{2}\right)\left\|u_{1}\right\|_{H^{1}(\Omega)}\left\|u_{2}\right\|_{H^{1}(\Omega)} .
\end{gathered}
$$

The most difficult step is to prove stability for the electric potentials. One is again faced with the problem of isolating the term containing $q_{1}-q_{2}$, only controlling the difference of the magnetic fields $d A_{1}-d A_{2}$. A natural idea is then to mimic the uniqueness proof, use the gauge invariance of the Cauchy data sets in the ball $B$ to modify the integral estimate above and plug in appropriate CGOs. More precisely, use $B$ instead of $\Omega$, replace $A_{2}$ by $A_{2}+\nabla \varphi$, for a $\varphi$ in $W^{1, \infty}(B)$ with $\left.\varphi\right|_{\partial B}=0$, and plug in CGOs for $L_{A_{1}, q_{1}}^{B} u_{1}=0$ and $L_{\overline{A_{2}+\nabla \varphi}, \overline{q_{2}}}^{B} u_{2}=0$. The crucial point here is that the $A_{1}-A_{2}$ term, that we cannot hope to control due to the non uniqueness of the magnetic potentials, is replaced by $A_{1}-\left(A_{2}+\nabla \varphi\right)$ in the integral estimate. This later term can be controlled by the difference $d A_{1}-d A_{2}$. One does this by choosing $\varphi$

\footnotetext{
2 Recently Haberman and Tataru proved in 13 uniqueness for the Calderón problem with continuously differentiable conductivities. The reason why their argument does not provide uniqueness for general Lipschitz conductivities is because, in the construction of the CGOs, they required to approximate the gradient of conductivities in $L^{\infty}$ sense.

3 The space $W^{1, p}(B)$ is the first-order Sobolev space based on $L^{p}$ with $p \in$ $[1,+\infty)$ or $p=\infty$.
} 
suitably so that one is able to derive the estimate

$$
\left\|A_{1}-\left(A_{2}+\nabla \varphi\right)\right\|_{L^{2}\left(B ; \mathbb{C}^{n}\right)} \lesssim\left\|d A_{1}-d A_{2}\right\|_{H^{-1} \Omega^{2}(B)}
$$

where $\varphi \in W^{1, \infty}(B)$ for which $\left.\varphi\right|_{\partial B}=0$. An appropriate choice for $\varphi$ is the exact component of the Hodge decomposition $A_{1}-A_{2}$, which vanishes on $\partial B$. It should be mentioned here that this is also roughly the idea in [25], which deals with the case of more regular potentials. This idea needs however several modifications to work in the less regular framework. The main reason for the need to carry out these modifications is that the estimate we are able to prove only holds for $\varphi$ in $W^{1, p}(B)$ for every $p \geq n$. The restriction $p \neq \infty$ is consequence of the elliptic regularity, which only holds for $1<p<+\infty$. Thus, if we did not modify the previous approach, we could not use Krupchyk and Uhlmann's method to construct CGOs for $L \frac{B}{A_{2}+\nabla \varphi, \overline{q_{2}}} u_{2}=0$ since $\varphi \notin W^{1, \infty}(B)$. Finally, let us point out that proving (11) becomes in our case more technical than in 25] due to to the lack of regularity. The argument in [25] is based on the open mapping theorem and it is enough to prove the bijectivity of certain operator - which is a qualitative property. Our approach is however based on the $H^{1}$ ellipticity of the Hodge Laplacian and a compactness argument.

The paper is organized as follows. In Section 2 we prove the integral estimate that will be used as the starting point of our argument. In Section 3 we review the construction of the CGOs due to Krupchyk and Uhlmann for the special case where the magnetic potentials satisfy a prescribed $L^{2}$ modulus of continuity of Hölder type. In Section 4 and Section 5 we prove stability for the magnetic fields and the electric potentials respectively. In Section 6 we prove estimate (1), which is the key ingredient in the proof of the stability for the electric potentials.

\section{From the BOUNDARY TO THE INTERIOR}

In this section we prove an integral estimate relating the electric and magnetic potentials in $\Omega$ with the distance between their corresponding Cauchy data sets. This integral estimate will be our starting point in proving the stability estimates for the IBVP under consideration.

Proposition 2.1. Let $A_{1}, A_{2} \in L^{\infty}\left(\Omega ; \mathbb{C}^{n}\right)$ be two magnetic potentials and let $q_{1}, q_{2} \in L^{\infty}(\Omega)$ be two electric potentials. Let $C_{j}$ with $j \in\{1,2\}$ denote the Cauchy data set associated to the operator $L_{A_{j}, q_{j}}$. Then, for any $u_{1} \in H^{1}(\Omega)$ solving $L_{A_{1}, q_{1}} u_{1}=0$ and any $u_{2} \in H^{1}(\Omega)$ solving $L_{\overline{A_{2}}, \overline{q_{2}}} u_{2}=0$, we have that

$$
\begin{aligned}
&\left|\int_{\Omega} i\left(A_{1}-A_{2}\right) \cdot\left(u_{1} \nabla \overline{u_{2}}-\overline{u_{2}} \nabla u_{1}\right)+\left(A_{1}^{2}-A_{2}^{2}+q_{1}-q_{2}\right) u_{1} \overline{u_{2}} d x\right| \\
& \lesssim \operatorname{dist}\left(C_{1}, C_{2}\right)\left[1+\left\|A_{2}\right\|_{L^{\infty}\left(\Omega ; \mathbb{C}^{n}\right)}^{2}+\left\|q_{2}\right\|_{L^{\infty}(\Omega)}\right]\left\|u_{1}\right\|_{H^{1}(\Omega)}\left\|u_{2}\right\|_{H^{1}(\Omega)},
\end{aligned}
$$

where the implicit constant is universal. 
Proof. Note that

$$
\begin{gathered}
\left\langle N_{A_{1}, q_{1}}^{U} u_{1}, \overline{T_{U} u_{2}}\right\rangle-\overline{\left\langle N \overline{A_{2}}, \overline{q_{2}} u_{2}, \overline{T_{U} u_{1}}\right\rangle} \\
=\int_{U} i\left(A_{1}-A_{2}\right) \cdot\left(u_{1} \nabla \overline{u_{2}}-\overline{u_{2}} \nabla u_{1}\right)+\left(A_{1}^{2}-A_{2}^{2}+q_{1}-q_{2}\right) u_{1} \overline{u_{2}} d x
\end{gathered}
$$

for any domain $U \subset \mathbb{R}^{n}$. In this proof we only use the case $U=\Omega$. For the same reason, we know that

$$
\left\langle\left\|T u_{1}\right\|_{T H^{1}(\Omega)} g_{2}, \overline{T u_{2}}\right\rangle-\overline{\left\langle N_{\overline{A_{2}}, \overline{q_{2}}} u_{2}, \overline{\left\|T u_{1}\right\|_{T H^{1}(\Omega)} f_{2}}\right\rangle}=0
$$

holds for every $\left(f_{2}, g_{2}\right) \in C_{A_{2}, q_{2}}$. Last identity immediately imply

$$
\begin{gathered}
\quad\left|\left\langle N_{A_{1}, q_{1}} u_{1}, \overline{T u_{2}}\right\rangle-\overline{\left\langle N_{\overline{A_{2}}, \overline{q_{2}}} u_{2}, \overline{T u_{1}}\right\rangle}\right| \\
\leq\left\|N_{A_{1}, q_{1}} u_{1}-\right\| T u_{1}\left\|_{T H^{1}(\Omega)} g_{2}\right\|_{T H^{1}(\Omega)^{*}}\left\|T u_{2}\right\|_{T H^{1}(\Omega)} \\
+\left\|N_{\overline{A_{2}}, \overline{q_{2}}} u_{2}\right\|_{T H^{1}(\Omega)^{*}}\left\|T u_{1}-\right\| T u_{1}\left\|_{T H^{1}(\Omega)} f_{2}\right\|_{T H^{1}(\Omega)} .
\end{gathered}
$$

On the other hand

$$
\left\|N_{\overline{A_{2}}, \overline{q_{2}}} u_{2}\right\|_{T H^{1}(\Omega)^{*}} \lesssim\left[1+\left\|A_{2}\right\|_{L^{\infty}\left(\Omega ; \mathbb{C}^{n}\right)}^{2}+\left\|q_{2}\right\|_{L^{\infty}(\Omega)}\right]\left\|u_{2}\right\|_{H^{1}(\Omega)} .
$$

Since

$$
\left\|T u_{j}\right\|_{T H^{1}(\Omega)} \leq\left\|u_{j}\right\|_{H^{1}(\Omega)}
$$

for $j \in\{1,2\}$, we have that

$$
\begin{aligned}
& \left|\left\langle N_{A_{1}, q_{1}} u_{1}, \overline{T u_{2}}\right\rangle-\overline{\left\langle N_{\overline{A_{2}}, \overline{q_{2}}} u_{2}, \overline{T u_{1}}\right\rangle}\right| \lesssim I\left(\left(f_{1}, g_{1}\right) ; C_{A_{2}, q_{2}}\right) \\
& \quad \times\left[1+\left\|A_{2}\right\|_{L^{\infty}\left(\Omega ; \mathbb{C}^{n}\right)}^{2}+\left\|q_{2}\right\|_{L^{\infty}(\Omega)}\right]\left\|u_{1}\right\|_{H^{1}(\Omega)}\left\|u_{2}\right\|_{H^{1}(\Omega)} .
\end{aligned}
$$

where

$$
f_{1}=\frac{T u_{1}}{\left\|T u_{1}\right\|_{T H^{1}(\Omega)}}, \quad g_{1}=\frac{N_{A_{1}, q_{1}} u_{1}}{\left\|T u_{1}\right\|_{T H^{1}(\Omega)}} .
$$

Now the statement of the proposition follows easily using (2) and taking supremum and then maximum.

\section{Complex GeOmetric optics SOlutions}

In this section, we review the properties of the CGOs constructed by Krupchyk and Uhlmann in [15] for the particular case where the magnetic potential satisfies a prescribed $L^{2}$-modulus of continuity. The additional regularity allows us to attain appropriate remainder estimates that are needed later. We end the section by estimating the $H^{1}$-norm of these CGOs.

Throughout this section we assume that $q \in L^{\infty}(U), A \in L^{\infty}\left(\mathbb{R}^{n} ; \mathbb{C}^{n}\right)$ and

$$
\operatorname{supp} A \subset \bar{U},
$$


where $U \subset \mathbb{R}^{n}$ is a domain. For notational convenience, we write throughout this section $\|q\|_{L^{\infty}}$ and $\|A\|_{L^{\infty}}$ to denote the norms of $q \in$ $L^{\infty}(U)$ and $A \in L^{\infty}\left(\mathbb{R}^{n} ; \mathbb{C}^{n}\right)$, respectively. In addition, we assume that $|A|_{B_{\varepsilon}^{2, \infty}}^{2}<\infty$. The definition of $|\cdot|_{B_{\varepsilon}^{2, \infty}}$ was given in Section 1 .

Let $\Psi$ belong to $C_{0}^{\infty}\left(\mathbb{R}^{n}\right)$ with $0 \leq \Psi(x) \leq 1$ for all $x \in \mathbb{R}^{n}$, supp $\Psi \subset$ $\left\{x \in \mathbb{R}^{n}:|x| \leq 1\right\}$ and $\int_{\mathbb{R}^{n}} \Psi d x=1$. Define $\Psi_{\tau}(x)=\tau^{-n} \Psi(x / \tau)$ for $\tau \in(0,1]$ and $x \in \mathbb{R}^{n}$. Then $A^{\sharp}=\Psi_{\tau} * A \in C_{0}^{\infty}\left(\mathbb{R}^{n}, \mathbb{C}^{n}\right)$ (where convolution is taken with each component of $A$ ) and $A^{b}=A-A^{\sharp}$ satisfies

$$
\left\|A^{b}\right\|_{L^{2}\left(\mathbb{R}^{n} ; \mathbb{C}^{n}\right)} \leq \tau^{\varepsilon}|A|_{B_{\varepsilon}^{2, \infty}}
$$

for $\tau \in(0,1]$. On the other hand,

$$
\left\|\partial^{\alpha} A^{\sharp}\right\|_{L^{\infty}\left(\mathbb{R}^{n} ; \mathbb{C}^{n}\right)} \lesssim \tau^{-|\alpha|}\|A\|_{L^{\infty}}
$$

for $\tau \in(0,1]$ and $\alpha \in \mathbb{N}^{n}$, where the implicit constant in this inequality only depends on $\Psi$.

In [15], Krupchyk and Uhlmann proved the existence of CGOs in $H^{1}(U)$ solving

$$
L_{A, q} u=0
$$

with $A \in L^{\infty}\left(U ; \mathbb{C}^{n}\right)$ and $q \in L^{\infty}(U)$. These CGOs are solutions of the form

$$
u(x ; \zeta, h)=e^{x \cdot \zeta / h}(a(x ; \zeta, h)+r(x ; \zeta, h))
$$

where $\zeta \in \mathbb{C}^{n}$ with $\zeta \cdot \zeta=0$ and $|\zeta| \sim 1 ; h$ is a small positive parameter; $a$ is a smooth amplitude and $r$ is a correction term. In the next lines we follow Krupchyk and Uhlmann's ideas to check the properties of $u(\cdot ; \zeta, h)$ in the particular case where $A$ and $q$ are as the beginning of the section.

Let the restriction of $A$ to $U$ be also denoted by $A$. Consider $\zeta=$ $\zeta_{0}+\zeta_{1}$ with $\zeta_{0}$ independent of $h, \operatorname{Re} \zeta_{0} \cdot \operatorname{Im} \zeta_{0}=0,\left|\operatorname{Re} \zeta_{0}\right|=\left|\operatorname{Im} \zeta_{0}\right|=1$ and $\left|\zeta_{1}\right|=\mathscr{O}(h)$ as $h$ becomes small. In order to construct $u(\cdot ; \zeta, h)$ of the form of (5) satisfying $L_{A, q} u=0$, it is enough to prove the existence of a $r(\cdot ; \zeta, h, \tau) \in H^{1}(U)$ solving

$$
e^{-\zeta \cdot(\cdot) / h} h^{2} L_{A, q}\left(e^{\zeta \cdot(\cdot) / h} r\right)=e^{-\zeta \cdot(\cdot) / h} h^{2} L_{A, q}\left(e^{\zeta \cdot(\cdot) / h} a\right)=: w,
$$

in $\mathbb{R}^{n}$. One does this by first finding an $a\left(\cdot ; \zeta_{0}, \tau\right) \in C^{\infty}\left(\mathbb{R}^{n}\right)$ that solves

$$
\zeta_{0} \cdot \nabla a+i \zeta_{0} \cdot A^{\sharp} a=0,
$$

so that $w$ becomes

$$
\begin{aligned}
w= & h^{2} \Delta a+i h^{2} A \cdot \nabla a-h^{2} m_{A}(a)-h^{2}\left(A^{2}+q\right) a+2 h \zeta_{1} \cdot \nabla a \\
& +2 h i \zeta_{0} \cdot A^{b} a+2 h i \zeta_{1} \cdot A a,
\end{aligned}
$$


where $m_{A}$ denotes the bounded linear operator from $H^{1}(U)$ to $H^{-1}(U)$ defined by

$$
\left\langle m_{A}(\phi), \psi\right\rangle=\int_{U} i \phi A \cdot \nabla \psi d x
$$

for all $\phi \in H^{1}(U)$ and all $\psi \in H_{0}^{1}(U)$.

If we look for solutions to (7) in the form

$$
a\left(\cdot ; \zeta_{0}, \tau\right)=e^{\Phi^{\sharp}\left(\cdot ; \zeta_{0}, \tau\right)},
$$

it will be enough that $\Phi^{\sharp}\left(\cdot ; \zeta_{0}, \tau\right)$ satisfies

$$
\zeta_{0} \cdot \nabla \Phi^{\sharp}+i \zeta_{0} \cdot A^{\sharp}=0
$$

in $\mathbb{R}^{n}$. Since $\operatorname{Re} \zeta_{0} \cdot \operatorname{Im} \zeta_{0}=0$ and $\left|\operatorname{Re} \zeta_{0}\right|=\left|\operatorname{Im} \zeta_{0}\right|=1, \zeta_{0} \cdot \nabla$ is a $\bar{\partial}$ operator in suitable coordinates. Therefore, $\Phi^{\sharp}=\left(\zeta_{0} \cdot \nabla\right)^{-1}\left(-i \zeta_{0} \cdot A^{\sharp}\right)$ belongs to $C^{\infty}\left(\mathbb{R}^{n}\right)$ and, using (4), we have that

$$
\left\|\partial^{\alpha} \Phi^{\sharp}\right\|_{L^{\infty}\left(\mathbb{R}^{n}\right)} \lesssim \tau^{-|\alpha|}\|A\|_{L^{\infty}}
$$

for $\tau \in(0,1]$ and $\alpha \in \mathbb{N}^{n}$ (For more details see Lemma 4.6 in [18] and Lemma 2.1 in [21]). Here the implicit constant only depends on $\alpha$. Moreover, $\Phi\left(\cdot ; \zeta_{0}\right)=\left(\zeta_{0} \cdot \nabla\right)^{-1}\left(-i \zeta_{0} \cdot A\right) \in L^{\infty}\left(\mathbb{R}^{n}\right)$ solves

$$
\zeta_{0} \cdot \nabla \Phi+i \zeta_{0} \cdot A=0
$$

and satifies

$$
\begin{aligned}
& \left\|\Phi\left(\cdot ; \zeta_{0}\right)\right\|_{L^{\infty}\left(\mathbb{R}^{n}\right)} \lesssim\|A\|_{L^{\infty}}, \\
& \left\|\chi\left(\Phi^{\sharp}\left(\cdot, \zeta_{0}, \tau\right)-\Phi\left(\cdot ; \zeta_{0}\right)\right)\right\|_{L^{2}\left(\mathbb{R}^{n}\right)} \lesssim \tau^{\varepsilon}|A|_{B_{\varepsilon}^{2, \infty}},
\end{aligned}
$$

for any $\chi \in C_{0}^{\infty}\left(\mathbb{R}^{n}\right)$. The implicit constant in (12) depends on $\chi$ and $U$. The estimate (12) is an immediate consequence of Lemma $3.1 \mathrm{in}$ [22] and the estimate (3).

Regarding equation (6), Krupchyk and Uhlmann proved (see Proposition 2.3 in [15]) that there exists a positive decreasing function $h_{0}$ defined in $(0,+\infty) \subset \mathbb{R}$ such that, for all $h \leq h_{0}\left(\|A\|_{L^{\infty}\left(U ; \mathbb{C}^{n}\right)}\right)$, there exists $r(\cdot ; \zeta, h, \tau)$ which is a $H^{1}(U)$ solution to (6) and satisfies

$$
\|r\|_{H_{\mathrm{scl}}^{1}(U)} \lesssim \frac{1}{h}\|w\|_{H_{\mathrm{scl}}^{-1}(U)} .
$$

Here the implicit constant depends on $U$. The semi-classical norms are defined by

$$
\begin{aligned}
\|r\|_{H_{\mathrm{scl}}^{1}(U)}^{2} & =\|r\|_{L^{2}(U)}^{2}+\|h \nabla r\|_{L^{2}\left(U ; \mathbb{C}^{n}\right)}^{2}, \\
\|w\|_{H_{\mathrm{scl}}^{-1}(U)} & =\sup _{\phi \in H_{0}^{1}(U) \backslash\{0\}} \frac{|\langle w, \phi\rangle|}{\|\phi\|_{H_{\mathrm{scl}}^{1}(U)}} .
\end{aligned}
$$

On of the key properties of a CGO solution is that the correction term $r$ tends to vanish, in some sense, when the parameter $h$ becomes small. This can be deduced from (13) by computing $\|w\|_{H_{\mathrm{scl}}^{-1}(U)}$ and choosing $\tau$ as a proper power of $h$ : 
By the Cauchy-Schwarz inequality and estimate (9) we can prove that there exists a constant $c>0$ such that, for any $\phi \in H_{0}^{1}(U)$,

$$
\begin{gathered}
\left|\left\langle h^{2} \Delta a+i h^{2} A \cdot \nabla a+2 h \zeta_{1} \cdot \nabla a+2 h i \zeta_{1} \cdot A a, \phi\right\rangle\right| \\
\lesssim \frac{h^{2}}{\tau^{2}} e^{c\|A\|_{L^{\infty}}}\left(1+\|A\|_{L^{\infty}}^{2}\right)\|\phi\|_{H_{\mathrm{scl}}^{1}(U)} .
\end{gathered}
$$

Again by the Cauchy-Schwarz inequality and (9), there exists a constant $c>0$ such that, for any $u \in H_{0}^{1}(U)$,

$$
\left|\left\langle h^{2}\left(A^{2}+q\right) a, \phi\right\rangle\right| \lesssim h^{2}\left(\|A\|_{L^{\infty}}^{2}+\|q\|_{L^{\infty}}\right) e^{c\|A\|_{L^{\infty}}}\|\phi\|_{H_{\mathrm{scl}}^{1}(U)} .
$$

By the Cauchy-Schwarz inequality and the estimates (3) and (9), there exists $c>0$ such that, for any $\phi \in H_{0}^{1}(U)$,

$$
\left|\left\langle 2 h i \zeta_{0} \cdot A^{b} a, \phi\right\rangle\right| \lesssim h \tau^{\varepsilon} e^{c\|A\|_{L} \infty}|A|_{B_{\varepsilon}^{2, \infty}}\|\phi\|_{H_{\mathrm{scl}}^{1}(U)} .
$$

Finally, by integrating by parts, Cauchy-Schwarz inequality, estimate (9)), (44) and (3), there exists a $c>0$ such that, for any $\phi \in H_{0}^{1}(U)$,

$$
\begin{aligned}
\left|\left\langle h^{2} m_{A}(a), \phi\right\rangle\right| & \leq\left|\int_{U} i h^{2} \nabla \cdot\left(a A^{\sharp}\right) \phi d x\right|+\left|\int_{U} i h^{2} a A^{b} \cdot \nabla \phi d x\right| \\
& \lesssim e^{c\|A\|_{L^{\infty}}}\left[\frac{h^{2}}{\tau}+\frac{h^{2}\|A\|_{L^{\infty}}^{2}}{\tau}+h \tau^{\varepsilon}|A|_{B_{\varepsilon}^{2, \infty}}\right]\|\phi\|_{H_{\mathrm{scl}}^{1}(U)} .
\end{aligned}
$$

The implicit constant in the last four inequalities depends on $U$. Therefore, choosing $\tau=h^{1 /(\varepsilon+2)}$ in the above estimates and using (13), we see that, for $h \leq h_{0}\left(\|A\|_{L^{\infty}\left(U ; \mathbb{C}^{n}\right)}\right)$,

$$
\|r\|_{H_{\mathrm{scl}}^{1}(U)} \lesssim h^{\frac{\varepsilon}{\varepsilon+2}} e^{c\|A\|_{L^{\infty}}}\left[1+\|A\|_{L^{\infty}}^{2}+\|q\|_{L^{\infty}}+|A|_{B_{\varepsilon}^{2, \infty}}\right] .
$$

We end this section by estimating the $H^{1}(U)$-norm of $u(\cdot ; \zeta, h)$ :

$$
\|u\|_{H^{1}(U)} \lesssim e^{c^{\prime} / h} e^{c\|A\|_{L^{\infty}}}\left[1+\|A\|_{L^{\infty}}^{2}+\|q\|_{L^{\infty}}+|A|_{B_{\varepsilon}^{2, \infty}}\right]
$$

where $c^{\prime}>0$ and the implicit constant depend on $U$.

\section{Stability estimates for the Magnetic FIELDS}

The aim of this section is to prove Theorem 1.1 by deriving the two stability estimates for the magnetic fields. The first step will be to use Proposition 2.1 and the CGOs constructed in Section 3 to estimate the Fourier transform of the difference of the magnetic fields. Then, we prove the stability estimates in Sobolev (the general approach follows [1]) and Besov spaces.

Consider an a priori constant $M \in[1,+\infty)$ and a small constant $\varepsilon \in(0,1)$. Let $A_{1}, A_{2} \in L^{\infty}\left(\Omega ; \mathbb{C}^{n}\right)$ be two magnetic potentials and let $q_{1}, q_{2} \in L^{\infty}(\Omega)$ be two electric potentials. Assume that the extension by zero of $A_{j}$ out of $\Omega$, still denoted by $A_{j}$, satisfies $\left|A_{j}\right|_{B_{\varepsilon}^{2, r}}<\infty$ with $r \in[1,+\infty)$ or $r=\infty$. Furthermore, assume that

$$
\left\|A_{j}\right\|_{L^{\infty}\left(\mathbb{R}^{n} ; \mathbb{C}^{n}\right)}+\left|A_{j}\right|_{B_{\varepsilon}^{2, r}}+\left\|q_{j}\right\|_{L^{\infty}(\Omega)} \leq M
$$


for $j \in\{1,2\}$. The implicit constants in the inequalities may, throughout this section, depend on $M$ and $\varepsilon$, as well as on $n$ and $\Omega$.

For any $\xi \in \mathbb{R}^{n}$, consider $\mu_{1}, \mu_{2} \in \mathbb{R}^{n}$ such that $\left|\mu_{1}\right|=\left|\mu_{2}\right|=1$ and $\mu_{1} \cdot \mu_{2}=\mu_{1} \cdot \xi=\mu_{2} \cdot \xi=0$. For any positive $h$ with $h \leq \min (1,2 /|\xi|)$, we define

$$
\begin{aligned}
& \zeta_{1}=\frac{i h \xi}{2}+\mu_{1}+i \sqrt{1-h^{2} \frac{|\xi|^{2}}{4}} \mu_{2} \\
& \zeta_{2}=-\frac{i h \xi}{2}-\mu_{1}+i \sqrt{1-h^{2} \frac{|\xi|^{2}}{4}} \mu_{2} .
\end{aligned}
$$

Note that $\zeta_{j} \cdot \zeta_{j}=0$ for $j \in\{1,2\}$ and $\left(\zeta_{1}+\overline{\zeta_{2}}\right) / h=i \xi$. Moreover, $\zeta_{1}=\mu_{1}+i \mu_{2}+\mathscr{O}(h)$ and $\zeta_{2}=-\mu_{1}+i \mu_{2}+\mathscr{O}(h)$.

Let

$$
u_{1}\left(x ; \zeta_{1}, h\right)=e^{x \cdot \zeta_{1} / h}\left(e^{\Phi_{1}^{\sharp}\left(x ; \mu_{1}+i \mu_{2}, h\right)}+r_{1}\left(x ; \zeta_{1}, h\right)\right)
$$

and

$$
u_{2}\left(x ; \zeta_{2}, h\right)=e^{x \cdot \zeta_{2} / h}\left(e^{\Phi_{2}^{\sharp}\left(x ;-\mu_{1}+i \mu_{2}, h\right)}+r_{2}\left(x ; \zeta_{2}, h\right)\right)
$$

be CGO solutions of $L_{A_{1}, q_{1}} u_{1}=0$ and $L_{\overline{A_{2}}, \overline{q_{2}}} u_{2}=0$-constructed $\sqrt{4}$ as in Section 3 for $U=\Omega$ and $\tau=h^{1 /(\varepsilon+2)}$.

We now state the estimate for the Fourier transform of the difference of the magnetic fields. Notice that we use the notations $A_{1}$ and $A_{2}$ for both the vector fields and the corresponding 1-forms, depending on the context.

Lemma 4.1. There exists a constant $c>0$ depending on $\Omega$ such that

$$
\left|\widehat{d A_{1}}(\xi)-\widehat{d A_{2}}(\xi)\right| \lesssim|\xi|\left(\operatorname{dist}\left(C_{1}, C_{2}\right) e^{c / h}+h^{\varepsilon /(\varepsilon+2)}\right)
$$

for all $h \leq \min \left(1,2 /|\xi|, h_{0}(M)\right)$.

Proof. To prove the statement we just need to plug in $u_{1}$ and $u_{2}$, as in (18) and (19), in the estimate of Proposition 2.1 multiplied by $h$ and then study the behaviour in $h$. The term $u_{1} \overline{u_{2}}$ is bounded in $h$, since $\left(\zeta_{1}+\overline{\zeta_{2}}\right) / h=i \xi$. One sees then by the Cauchy-Schwarz inequality, (9) and (14), that

$$
\left|\int_{\Omega}\left(A_{1}^{2}-A_{2}^{2}+q_{1}-q_{2}\right) u_{1} \overline{u_{2}} d x\right| \lesssim 1 .
$$

Therefore, by Proposition [2.1, (15) and (20), there exists a constant $c>0$, that depends on $\Omega$, such that

$$
h\left|\int_{\Omega}\left(A_{1}-A_{2}\right) \cdot\left(u_{1} \nabla \overline{u_{2}}-\overline{u_{2}} \nabla u_{1}\right) d x\right| \lesssim \operatorname{dist}\left(C_{1}, C_{2}\right) e^{c / h}+h .
$$

\footnotetext{
${ }^{4}$ Note that the bounded inclusion $B_{\varepsilon}^{2, r}\left(\mathbb{R}^{n}\right) \hookrightarrow B_{\varepsilon}^{2, \infty}\left(\mathbb{R}^{n}\right)$ and (16) provide an a priori bound for $\left|A_{j}\right|_{B_{\varepsilon}^{2, \infty}}$ depending on $M$.
} 
Again by Cauchy-Schwarz inequality, (9) and (14); one can estimate the left hand side of last estimate from bellow as follows

$$
\begin{aligned}
& \left|\int_{\Omega}\left(A_{1}-A_{2}\right) \cdot\left(\overline{\zeta_{2}}-\zeta_{1}\right) e^{i x \cdot \xi} e^{\Phi_{1}^{\sharp}+\overline{\Phi_{2}^{\sharp}}} d x\right| \\
& \quad \lesssim h\left|\int_{\Omega}\left(A_{1}-A_{2}\right) \cdot\left(u_{1} \nabla \overline{u_{2}}-\overline{u_{2}} \nabla u_{1}\right) d x\right|+h^{\varepsilon /(\varepsilon+2)} .
\end{aligned}
$$

Now we want to replace $\Phi_{j}^{\sharp}$ by $\Phi_{j}$ on the right hand side of this estimate. Since $\overline{\zeta_{2}}-\zeta_{1}=-2\left(\mu_{1}+i \mu_{2}\right)+\mathscr{O}(h)$ we have, by (9) $)$, that

$$
\begin{aligned}
& \left|\int_{\Omega}\left(A_{1}-A_{2}\right) \cdot\left(\mu_{1}+i \mu_{2}\right) e^{i x \cdot \xi} e^{\Phi_{1}+\overline{\Phi_{2}}} d x\right| \\
& \quad \lesssim\left|\int_{\Omega}\left(A_{1}-A_{2}\right) \cdot\left(\overline{\zeta_{2}}-\zeta_{1}\right) e^{i x \cdot \xi} e^{\Phi_{1}^{\sharp}+\overline{\Phi_{2}^{\sharp}}} d x\right| \\
& \quad+\left|\int_{\Omega}\left(A_{1}-A_{2}\right) \cdot\left(\mu_{1}+i \mu_{2}\right) e^{i x \cdot \xi}\left(e^{\Phi_{1}^{\sharp}+\overline{\Phi_{2}^{\sharp}}}-e^{\Phi_{1}+\overline{\Phi_{2}}}\right) d x\right|+h .
\end{aligned}
$$

The second integral on the right hand side can be estimated as

$$
\left|\int_{\Omega}\left(A_{1}-A_{2}\right) \cdot\left(\mu_{1}+i \mu_{2}\right) e^{i x \cdot \xi}\left(e^{\Phi_{1}^{\sharp}+\overline{\Phi_{2}^{\sharp}}}-e^{\Phi_{1}+\overline{\Phi_{2}}}\right) d x\right| \lesssim h^{\varepsilon /(\varepsilon+2)} .
$$

using (12), (9), (11) and the inequality

$$
\left|e^{z_{1}}-e^{z_{2}}\right| \leq\left|z_{1}-z_{2}\right| e^{\max \left(\operatorname{Re} z_{1}, \operatorname{Re} z_{2}\right)} .
$$

Thus, we may write

$$
\left|\left(\mu_{1}+i \mu_{2}\right) \cdot \int_{\Omega}\left(A_{1}-A_{2}\right) e^{i x \cdot \xi} e^{\Phi_{1}+\overline{\Phi_{2}}} d x\right| \lesssim \operatorname{dist}\left(C_{1}, C_{2}\right) e^{c / h}+h^{\varepsilon /(\varepsilon+2)} .
$$

Now by Proposition 3.3 in [15] we can remove $e^{\Phi_{1}+\overline{\Phi_{2}}}$ and get that

$$
\left|\left(\mu_{1}+i \mu_{2}\right) \cdot \int_{\Omega}\left(A_{1}-A_{2}\right) e^{i x \cdot \xi} d x\right| \lesssim \operatorname{dist}\left(C_{1}, C_{2}\right) e^{c / h}+h^{\varepsilon /(\varepsilon+2)} .
$$

To finish the proof, note that the above computations also hold if we replace $\mu_{1}+i \mu_{2}$ by $\mu_{1}-i \mu_{2}$, hence

$$
\left|\mu \cdot \int_{\Omega}\left(A_{1}-A_{2}\right) e^{i x \cdot \xi} d x\right| \lesssim \operatorname{dist}\left(C_{1}, C_{2}\right) e^{c / h}+h^{\varepsilon /(\varepsilon+2)}
$$

for any unit vector $\mu$ such that $\mu \cdot \xi=0$. In particular, it holds for the vectors $\mu_{j, k}=\left(\xi_{j}^{2}+\xi_{k}^{2}\right)^{-1 / 2}\left(\xi_{j} e_{k}-\xi_{k} e_{j}\right)$ with $j, k \in\{1, \ldots, n\}$, since $\mu_{j, k} \cdot \xi=0$. Here $\xi_{j}$ denotes the $j$-th component of $\xi$ and $e_{k}$ the $k$-th element of the canonical basis of $\mathbb{R}^{n}$. Thus

$$
\left|\widehat{d A_{1}}(\xi)-\widehat{d A_{2}}(\xi)\right| \lesssim|\xi|\left(\operatorname{dist}\left(C_{1}, C_{2}\right) e^{c / h}+h^{\varepsilon /(\varepsilon+2)}\right)
$$


Next we derive the stability estimate for the difference of the magnetic fields in the Sobolev space $H^{-1} \Omega^{2}\left(\mathbb{R}^{n}\right)$ using the equivalent norm given by

$$
\|u\|_{H^{-1} \boldsymbol{\Omega}^{2}\left(\mathbb{R}^{n}\right)}^{2}=\int_{\mathbb{R}^{n}}\left(1+|\xi|^{2}\right)^{-1}|\widehat{u}(\xi)|^{2} d \xi .
$$

Proposition 4.2. There exist constants $c>1$ depending on $\Omega$ and $0<\tilde{c}<1$ universal such that

$$
\left\|d A_{1}-d A_{2}\right\|_{H^{-1} \Omega^{2}\left(\mathbb{R}^{n}\right)} \lesssim\left|\log \operatorname{dist}\left(C_{1}, C_{2}\right)\right|^{-\tilde{c} \varepsilon^{2} / n},
$$

provided that

$$
\left|\log \operatorname{dist}\left(C_{1}, C_{2}\right)\right|^{-1} \leq 1 / c \min \left(1, h_{0}(M)\right) .
$$

Proof. Let $B_{\rho}$ be denote the ball centred at $0 \in \mathbb{R}^{n}$ of radius $\rho \geq 1$ and let $B_{\rho}^{c}$ denote its complement in $\mathbb{R}^{n}$. Let $A$ denote $A_{1}-A_{2}$ for clarity. Using Lemma 4.1 we may estimate

$$
\int_{B_{\rho}} \frac{|\widehat{d A}(\xi)|^{2}}{1+|\xi|^{2}} d \xi \lesssim \rho^{n}\left(\operatorname{dist}\left(C_{1}, C_{2}\right) e^{c / h}+h^{\varepsilon /(\varepsilon+2)}\right)^{2}
$$

for all $h \leq \min \left(1,2 / \rho, h_{0}(M)\right)$. Note that this $c$ does not denote the one in the statement. On the other hand, write $A=A^{\sharp}+A^{b}$ using the same notation as in Section [3, where the parameter $\tau$ here is to be chosen. Then

$$
\begin{aligned}
\int_{B_{\rho}^{c}} \frac{|\widehat{d A}(\xi)|^{2}}{1+|\xi|^{2}} d \xi & \lesssim \int_{B_{\rho}^{c}} \frac{\mid \widehat{\left.d A^{\sharp}\right|^{2}}}{1+|\xi|^{2}} d \xi+\int_{B_{\rho}^{c}} \frac{|\xi|^{2}\left|\widehat{A^{b}}\right|^{2}}{1+|\xi|^{2}} d \xi \\
& \lesssim \rho^{-2}\left\|d A^{\sharp}\right\|_{L^{2} \boldsymbol{\Omega}^{2}\left(\mathbb{R}^{n}\right)}^{2}+\left\|A^{b}\right\|_{L^{2} \boldsymbol{\Omega}^{1}\left(\mathbb{R}^{n}\right)}^{2}
\end{aligned}
$$

Since the $\operatorname{supp} A$ is compact, estimates (4) and (3) imply that

$$
\int_{B_{\rho}^{c}} \frac{|\widehat{d A}(\xi)|^{2}}{1+|\xi|^{2}} d \xi \lesssim \rho^{-2} \tau^{-2}+\tau^{2 \varepsilon}
$$

Choosing $\tau=\rho^{-1 /(\varepsilon+1)}$, we have

$$
\|d A\|_{H^{-1} \boldsymbol{\Omega}^{2}\left(\mathbb{R}^{n}\right)}^{2} \lesssim \rho^{n} \operatorname{dist}\left(C_{1}, C_{2}\right)^{2} e^{2 c / h}+\rho^{n} h^{2 \varepsilon /(\varepsilon+2)}+\rho^{-2 \varepsilon /(\varepsilon+1)}
$$

by (22) and (23) . By equating the two last terms on the right hand side we express $\rho$ in terms of $h$ as

$$
\rho=h^{-\frac{2 \varepsilon(1+\varepsilon)}{(2+\varepsilon)(n+n \varepsilon+2 \varepsilon)}},
$$

which gives

$$
\|d A\|_{H^{-1} \boldsymbol{\Omega}^{2}\left(\mathbb{R}^{n}\right)}^{2} \lesssim \operatorname{dist}\left(C_{1}, C_{2}\right)^{2} e^{c^{\prime} / h}+h^{4 \varepsilon^{2} /((\varepsilon+2)(n+n \varepsilon+2 \varepsilon))}
$$

for $c^{\prime}>2 c$. Note that this choice of $\rho$ satisfies the restriction $\rho \leq 2 / h$. Finally, we set

$$
h=c^{\prime}\left|\log \operatorname{dist}\left(C_{1}, C_{2}\right)\right|^{-1}
$$

to prove the statement. 
We next derive the stability estimate for the difference of the magnetic fields in the Besov space $B_{-\delta}^{2, r} \Omega^{2}\left(\mathbb{R}^{n}\right)$ with $1>\delta>(1-\varepsilon)$ and the norm given by

$$
\|u\|_{B_{-\delta}^{2, r} \Omega^{2}\left(\mathbb{R}^{n}\right)}^{r}=\sum_{j \in \mathbb{N}} 2^{-r \delta j}\left\|\Delta_{j} u\right\|_{L^{2} \boldsymbol{\Omega}^{2}\left(\mathbb{R}^{n}\right)}^{r}
$$

for $r \in[1,+\infty)$ and

$$
\|u\|_{B_{-\delta}^{2, \infty} \boldsymbol{\Omega}^{2}\left(\mathbb{R}^{n}\right)}=\sup _{j \in \mathbb{N}}\left(2^{-\delta j}\left\|\Delta_{j} u\right\|_{L^{2} \boldsymbol{\Omega}^{2}\left(\mathbb{R}^{n}\right)}\right)
$$

for $r=\infty$.

In the following lines we describe the family of operators $\left\{\Delta_{j}\right\}_{j \in \mathbb{N}}$. We begin by picking a smooth cut-off function $\eta$ defined in $\mathbb{R}^{n}$ such that $\eta(\xi)=1$ for $|\xi| \leq 1$ and $\eta(\xi)=0$ for $|\xi| \geq 2$ and $\kappa$ being defined as $\kappa(\xi)=\eta(\xi)-\eta(2 \xi)$. Note that $\kappa$ is supported in the shell $\left\{\xi \in \mathbb{R}^{n}: 1 / 2 \leq|\xi| \leq 2\right\}$ and $\kappa\left(2^{-j} \cdot\right)$ is supported in $\left\{\xi \in \mathbb{R}^{n}\right.$ : $\left.2^{j-1} \leq|\xi| \leq 2^{j+1}\right\}$. Notice that it follows from the definitions that these functions form a partition of unity, i.e.

$$
1=\eta(\xi)+\sum_{j \in \mathbb{N} \backslash\{0\}} \kappa\left(2^{-j} \xi\right)
$$

for all $\xi \in \mathbb{R}^{n}$. Finally let $\psi_{0}$ be defined as $\widehat{\psi_{0}}(\xi)=\eta(\xi)$ and let $\psi_{j}$ with $j \in \mathbb{N} \backslash\{0\}$ be defined as $\widehat{\psi_{j}}(\xi)=\kappa\left(2^{-j} \xi\right)$. The operator $\Delta_{j}$ with $j \in \mathbb{N}$ is then defined as $\Delta_{j} u=\psi_{j} * u$.

Proposition 4.3. There exist constants $c>1$ depending on $\Omega$ and $0<\tilde{c}<1$ universal such that

$$
\left\|d A_{1}-d A_{2}\right\|_{B_{-\delta}^{2, r} \Omega^{2}\left(\mathbb{R}^{n}\right)} \lesssim\left|\log \operatorname{dist}\left(C_{1}, C_{2}\right)\right|^{-\tilde{c} \varepsilon(\delta-1+\varepsilon) / n},
$$

provided that

$$
\left|\log \operatorname{dist}\left(C_{1}, C_{2}\right)\right|^{-1} \leq 1 / c \min \left(1, h_{0}(M)\right) .
$$

The implicit constant above depends also on $\delta$.

Proof. Let $A$ denote $A_{1}-A_{2}$ for clarity. Consider $k \in \mathbb{N}$ to be chosen later. For any $j \in \mathbb{N}$ such that $j \leq k$ we have by Lemma 4.1 that

$$
2^{-\delta j}\left\|\Delta_{j} d A\right\|_{L^{2} \boldsymbol{\Omega}^{2}\left(\mathbb{R}^{n}\right)} \lesssim 2^{k(n / 2+1)}\left(\operatorname{dist}\left(C_{1}, C_{2}\right) e^{c / h}+h^{\varepsilon /(\varepsilon+2)}\right)
$$

for all $h \leq \min \left(2^{-k}, h_{0}(M)\right)$. Note that this $c$ does not denote the one in the statement. On the other hand, if $j>k$, then

since $\delta-1+\varepsilon>0$. Thus,

$$
2^{-\delta j}\left\|\Delta_{j} d A\right\|_{L^{2} \boldsymbol{\Omega}^{2}\left(\mathbb{R}^{n}\right)} \lesssim 2^{-k(\delta-1+\varepsilon)} 2^{j \varepsilon}\left\|\Delta_{j} A\right\|_{L^{2} \boldsymbol{\Omega}^{1}\left(\mathbb{R}^{n}\right)}
$$

$$
\|d A\|_{B_{-\delta}^{2, r} \Omega^{2}\left(\mathbb{R}^{n}\right)} \lesssim 2^{k n} \operatorname{dist}\left(C_{1}, C_{2}\right) e^{c / h}+2^{k n} h^{\varepsilon /(\varepsilon+2)}+2^{-k(\delta-1+\varepsilon)}
$$

for all $h \leq \min \left(2^{-k}, h_{0}(M)\right)$. Now choosing $k \in \mathbb{N}$ such that

$$
2^{-(k+1)}<h^{\frac{\varepsilon}{(\varepsilon+2)(\delta-1+\varepsilon+n)}} \leq 2^{-k}
$$


we know that there exists a constant $c^{\prime}>0$ such that

$$
\|d A\|_{B_{-\delta}^{2, \infty} \Omega^{2}\left(\mathbb{R}^{n}\right)} \lesssim \operatorname{dist}\left(C_{1}, C_{2}\right) e^{c^{\prime} / h}+h^{\frac{\varepsilon(\delta-1+\varepsilon)}{(\varepsilon+2)(\delta-1+\varepsilon+n)}} .
$$

Note that the choice of $k$ satisfies the restriction $h \leq 2^{-k}$. Finally, we set

$$
h=2 c^{\prime}\left|\log \operatorname{dist}\left(C_{1}, C_{2}\right)\right|^{-1}
$$

to prove the statement.

\section{Stability estimates For the EleCtRiC Potentials}

In this section we prove Theorem 1.2 by deriving the two stability estimates for the electric potentials. Our starting point could again be the estimate given in Proposition 2.1. There are however some difficulties with this. It seems that in order to isolate in that inequality the difference $q_{1}-q_{2}$ we would need to control the difference $A_{1}-A_{2}$. Unfortunately we can only control the difference of the magnetic fields $d A_{1}-d A_{2}$. To overcome this difficulty we give a slight modification of the estimate in Proposition 2.1. This modification is based on the invariance of the Cauchy data sets under gauge transformations in an open ball $B$ containing $\bar{\Omega}$ (see Lemma 5.1 below). Then, we use the CGOs constructed in Section 3 to estimate the Fourier transform of the difference of the electric potentials. Finally, we prove the stability estimates in Sobolev and Besov spaces.

As in the previous section we consider an a priori constant $M \in$ $[1,+\infty)$ and a small constant $\varepsilon \in(0,1)$. Let $A_{1}, A_{2} \in L^{\infty}\left(\Omega ; \mathbb{C}^{n}\right)$ be two magnetic potentials and let $q_{1}, q_{2} \in L^{\infty}(\Omega)$ be two electric potentials. Assume that the extension by zero of $A_{j}$ out of $\Omega$, still denoted by $A_{j}$, satisfies $\left|A_{j}\right|_{B_{\varepsilon}^{2, r}}<\infty$ with $r \in[1,+\infty)$ or $r=\infty$. Let $q_{1}$ and $q_{2}$ also denote the extensions by zero of the electric potentials. Furthermore, assume that

$$
\left\|A_{j}\right\|_{L^{\infty}\left(\mathbb{R}^{n} ; \mathbb{C}^{n}\right)}+\left|A_{j}\right|_{B_{\varepsilon}^{2, r}}+\left\|q_{j}\right\|_{L^{\infty}\left(\mathbb{R}^{n}\right)} \leq M
$$

for $j \in\{1,2\}$. Throughout this section, the constants implicit in each inequality may depend on $M$ and $\varepsilon$, as well as on $n, \Omega$ and on an open ball $B$ containing $\bar{\Omega}$.

For notational convenience, the norms $\|\cdot\|_{L^{p}(B)}$ and $\|\cdot\|_{L^{p}\left(B ; \mathbb{C}^{n}\right)}$ with $1 \leq p \leq \infty$ will be denoted by $\|\cdot\|_{L^{p}}$ and we will write $\left(A_{2}+\nabla \varphi\right)^{2}=$ $\left(A_{2}+\nabla \varphi\right) \cdot\left(A_{2}+\nabla \varphi\right)$.

Lemma 5.1. Let $B$ denote an open ball containing $\bar{\Omega}$ and let $\varphi$ belong to $W^{1, n}(B) \cap L^{\infty}(B)$ with $\left.\varphi\right|_{\partial B}=0$. Then, for any $u_{1}, u_{2} \in H^{1}(B)$ 
solving $L_{A_{1}, q_{1}}^{B} u_{1}=0$ and $L \frac{B}{\overline{A_{2}}, \overline{q_{2}}} u_{2}=0$, we have

$$
\begin{aligned}
& \mid \int_{B} i e^{i \varphi}\left(A_{1}-\left(A_{2}+\nabla \varphi\right)\right) \cdot\left(u_{1} \nabla \overline{u_{2}}-\overline{u_{2}} \nabla u_{1}\right) \\
& \quad+e^{i \varphi}\left(A_{1}^{2}-\left(A_{2}+\nabla \varphi\right)^{2}+q_{1}-q_{2}-\left(A_{1}-A_{2}-\nabla \varphi\right) \cdot \nabla \varphi\right) u_{1} \overline{u_{2}} d x \mid \\
& \quad \quad \lesssim \operatorname{dist}\left(C_{1}, C_{2}\right)\left\|u_{1}\right\|_{H^{1}(\Omega)}\left\|u_{2}\right\|_{H^{1}(\Omega)} .
\end{aligned}
$$

Proof. Since the restrictions of $u_{1}$ and $u_{2}$ to $\Omega$ (still denoted by $u_{1}$ and $u_{2}$ ) satisfy $L_{A_{1}, q_{1}}^{\Omega} u_{1}=0$ and $L_{\overline{A_{2}}, \overline{q_{2}}}^{\Omega} u_{2}=0$, we have, by Proposition 2.1, that

$$
\left|\int_{\Omega} i\left(A_{1}-A_{2}\right) \cdot\left(u_{1} \nabla \overline{u_{2}}-\overline{u_{2}} \nabla u_{1}\right)+\left(A_{1}^{2}-A_{2}^{2}+q_{1}-q_{2}\right) u_{1} \overline{u_{2}} d x\right|
$$

$$
\lesssim \operatorname{dist}\left(C_{1}, C_{2}\right)\left\|u_{1}\right\|_{H^{1}(\Omega)}\left\|u_{2}\right\|_{H^{1}(\Omega)} .
$$

Note that $A_{j}$ and $q_{j}$ have been extended as zero out of $\Omega$, so the domain of integration of the left hand side of (25) can be trivially augmented to $B$.

On the other hand, by identity (2), we know that

$$
\begin{aligned}
\int_{B} i\left(A_{1}\right. & \left.-A_{2}\right) \cdot\left(u_{1} \nabla \overline{u_{2}}-\overline{u_{2}} \nabla u_{1}\right)+\left(A_{1}^{2}-A_{2}^{2}+q_{1}-q_{2}\right) u_{1} \overline{u_{2}} d x \\
& =\left\langle N_{A_{1}, q_{1}}^{B} u_{1}, \overline{T_{B} u_{2}}\right\rangle-\overline{\left\langle N \frac{B}{A_{2}, \overline{q_{2}}} u_{2}, \overline{T_{B} u_{1}}\right\rangle} \\
& \left.\left.=\left\langle N_{A_{1}, q_{1}}^{B} u_{1}, \overline{T_{B} u_{2}}\right\rangle-\overline{\left\langle N \frac{B}{A_{2}+\nabla \varphi}, \overline{q_{2}}\right.}\left(e^{-i \bar{\varphi}} u_{2}\right), \overline{T_{B}\left(e^{-i \varphi} u_{1}\right.}\right)\right\rangle .
\end{aligned}
$$

The last identity is just a straightforward computation, which can be justified because $e^{-i \varphi} u_{1}$ and $e^{-i \bar{\varphi}} u_{2}$ belong to $H^{1}(B)$ and the last one satisfies

$$
L \frac{B}{A_{2}+\nabla \varphi, \overline{q_{2}}}\left(e^{-i \bar{\varphi}} u_{2}\right)=0 .
$$

The fact that $e^{-i \varphi} u_{1}$ and $e^{-i \bar{\varphi}} u_{2}$ belong to $H^{1}(B)$ can be deduced, by Sobolev's embeddings, from the following inequalities

$$
\begin{aligned}
& \left\|e^{-i \varphi} u_{1}\right\|_{H^{1}(B)} \lesssim e^{\|\varphi\|_{L^{\infty}}}\left(\left\|u_{1}\right\|_{L^{2}}+\|\nabla \varphi\|_{L^{n}}\left\|u_{1}\right\|_{L^{d}}+\left\|\nabla u_{1}\right\|_{L^{2}}\right) \\
& \left\|e^{-i \bar{\varphi}} u_{2}\right\|_{H^{1}(B)} \lesssim e^{\|\varphi\|_{L^{\infty}}}\left(\left\|u_{2}\right\|_{L^{2}}+\|\nabla \varphi\|_{L^{n}}\left\|u_{2}\right\|_{L^{d}}+\left\|\nabla u_{2}\right\|_{L^{2}}\right)
\end{aligned}
$$

where $d=2 n /(n-2)$. These estimates are consequences of Hölder inequality.

Since $\varphi$ vanishes on the boundary of $B$, we know that

$$
T_{B}\left(e^{-i \bar{\varphi}} u_{2}\right)=T_{B} u_{2}, \quad T_{B}\left(e^{-i \varphi} u_{1}\right)=T_{B} u_{1}
$$


(see for example Lemma 2 in [3]). Thus, again by identity (2) as well as (27), (26) and (25) we get

$$
\begin{aligned}
& \mid \int_{B} i\left(A_{1}-\left(A_{2}+\nabla \varphi\right)\right) \cdot\left(u_{1} \nabla\left(e^{i \varphi} \overline{u_{2}}\right)-e^{i \varphi} \overline{u_{2}} \nabla u_{1}\right) \\
& \quad+\left(A_{1}^{2}-\left(A_{2}+\nabla \varphi\right)^{2}+q_{1}-q_{2}\right) u_{1} e^{i \varphi} \overline{u_{2}} d x \mid \\
& \quad \lesssim \operatorname{dist}\left(C_{1}, C_{2}\right)\left\|u_{1}\right\|_{H^{1}(\Omega)}\left\|u_{2}\right\|_{H^{1}(\Omega)} .
\end{aligned}
$$

Now the integral term on last estimate can be rewritten as in the statement of the proposition.

The idea will be now to use the specific Hodge decomposition of Section 6 and write $A_{1}-A_{2}=d \psi+\delta F$, with the fact that we are able control the norm of the co-exact part $\delta F$, i.e. $\left\|A_{1}-\left(A_{2}+\nabla \psi\right)\right\|_{L^{2}}$ (see estimate (29) ). Lemma 5.1, allows us then to obtain an inequality with a gradient term added to $A_{1}-A_{2}$. By adding $\nabla \psi$ we would thus get an integral estimate with terms that we know how to control. We cannot however directly add $\nabla \psi$, because of the requirement that $\left.\varphi\right|_{\partial B}=0$ in Lemma 5.1. We resolve this problem by using a cut-off argument.

We choose $\varphi$ in Lemma 5.1 as $\varphi=\chi\left(\psi-\psi^{*}\right)$, where $\chi$ will be a smooth cut-off function, with $\chi=1$ on the supports of the potentials and such that it makes $\varphi$ vanish near $\partial B$ and $\psi^{*}$ is a constant. The idea of the cut-off argument is roughly to split $\nabla \psi$ as $\nabla \psi=\nabla(\chi \psi)+$ $\nabla((1-\chi) \psi)$. Since $\nabla(\chi \psi)=\nabla \varphi$, this part leads to terms that can be handled with Lemma 5.1. The support of the other part $\nabla((1-\chi) \psi)$ is disjoint from the supports of the potentials. But outside the supports of the potentials $d \psi=\delta F$. One can hence expect to be able to apply estimate (29). This is done by using the related estimate (30).

It might be helpful for the reader to know, prior to reading Section 6. that $\nabla \psi$ is the sum of the exact component of the Hodge decomposition of $A_{1}-A_{2}$ which vanishes on $\partial B$ and the exact expression of its harmonic component 5

Proposition 5.2. There exists $\psi \in W^{1, p}(B)$ with $p \geq 2$ satisfying the following conditions

$$
\|\psi\|_{W^{1, p}(B)} \lesssim\left\|A_{1}-A_{2}\right\|_{L^{p}}
$$

and

$$
\left\|A_{1}-\left(A_{2}+\nabla \psi\right)\right\|_{L^{2}} \lesssim\left\|d\left(A_{1}-A_{2}\right)\right\|_{H^{-1} \Omega^{2}(B)} .
$$

Moreover, if $B^{\prime}$ is a ball containing $\bar{\Omega}$ and such that $\overline{B^{\prime}} \subset B$, then

$$
\left\|\psi-\psi^{*}\right\|_{H^{1}\left(B \backslash \overline{B^{\prime}}\right)} \lesssim\left\|d\left(A_{1}-A_{2}\right)\right\|_{H^{-1} \Omega^{2}(B)},
$$

\footnotetext{
${ }^{5}$ In the more regular framework of [25], it was possible to take $\nabla \psi$ as the exact component of the Hodge decomposition of $A_{1}-A_{2}$ vanishing on $\partial B$. Thus, there was no need of introducing the cut-off function $\chi$ or controlling $\psi-\psi^{*}$ by $d A_{1}-d A_{2}$.
} 
where $\psi^{*}$ denotes the average of $\psi$ in $B \backslash \overline{B^{\prime}}$.

In order to continue with the argument, we postpone the proof of Proposition 5.2, which is given in Section 6.

In our analysis we will consider $\varphi=\chi\left(\psi-\psi^{*}\right)$ for $\chi \in C_{0}^{\infty}(B)$ such that $\chi(x)=1$ for all $x \in B^{\prime}$ and $p>n$. Thus,

$$
e^{\|\varphi\|_{L^{\infty}}}\left(1+\|\nabla \varphi\|_{L^{n}}+\|\nabla \psi\|_{L^{n}}\right) \lesssim 1
$$

by Morrey's inequality, (28) and the boundedness of $B$.

For any $\xi \in \mathbb{R}^{n}$ and $h \leq \min (1,2 /|\xi|)$, consider $\zeta_{1}$ and $\zeta_{2}$ as in (17). Let $u_{1}$ and $u_{2}$ be in the form (18) and (19) such that they solve $L_{A_{1}, q_{1}} u_{1}=0$ and $L_{\overline{A_{2}}, \overline{q_{2}}} u_{2}=0$-constructed 6 as in Section 3 for $U=B$ and $\tau=h^{1 /(\varepsilon+2)}$. We now state the estimate for the Fourier transform of the difference of the electric potentials by plugging in these solutions in the integral inequality given in Lemma 5.1.

Lemma 5.3. Let $\theta$ belong to $(0,2 / n) \subset \mathbb{R}$. There exist constants $0<\tilde{c}<1$ universal and $c>1$ depending on $\Omega, B, n$ and $\theta$ such that

$$
\begin{aligned}
\left|\widehat{q_{1}}(\xi)-\widehat{q_{2}}(\xi)\right| \lesssim & \operatorname{dist}\left(C_{1}, C_{2}\right) e^{c / h}+\left|\log \operatorname{dist}\left(C_{1}, C_{2}\right)\right|^{-\tilde{c} \theta \varepsilon^{2} / n} h^{-5 / 2} \\
& +h^{\varepsilon /(\varepsilon+2)}
\end{aligned}
$$

for all $h \leq \min \left(1,2 /|\xi|, h_{0}(c M)\right)$, provided that

$$
\left|\log \operatorname{dist}\left(C_{1}, C_{2}\right)\right|^{-1} \leq 1 / c \min \left(1, h_{0}(M)\right) .
$$

Note that the implicit constant above also depends on $\theta$.

Proof. Adding and subtracting the same terms we get that

$$
\begin{aligned}
& \left|\int_{B} e^{i \varphi}\left(q_{1}-q_{2}\right) u_{1} \overline{u_{2}} d x\right| \leq\left|\int_{B} e^{i \varphi}\left(A_{1}^{2}-\left(A_{2}+\nabla \psi\right)^{2}\right) u_{1} \overline{u_{2}} d x\right| \\
& +\left|\int_{B} i e^{i \varphi}\left(A_{1}-\left(A_{2}+\nabla \psi\right)\right) \cdot\left(u_{1} \nabla \overline{u_{2}}-\overline{u_{2}} \nabla u_{1}\right) d x\right| \\
& +\left|\int_{B} e^{i \varphi}\left(A_{1}-A_{2}-\nabla \psi\right) \cdot \nabla \varphi u_{1} \overline{u_{2}} d x\right|+I
\end{aligned}
$$

where $I$ denotes

$$
\begin{aligned}
& \mid \int_{B} i e^{i \varphi}\left(A_{1}-\left(A_{2}+\nabla \psi\right)\right) \cdot\left(u_{1} \nabla \overline{u_{2}}-\overline{u_{2}} \nabla u_{1}\right) \\
& \quad+e^{i \varphi}\left(A_{1}^{2}-\left(A_{2}+\nabla \psi\right)^{2}+q_{1}-q_{2}-\left(A_{1}-A_{2}-\nabla \psi\right) \cdot \nabla \varphi\right) u_{1} \overline{u_{2}} d x \mid .
\end{aligned}
$$

\footnotetext{
${ }^{6}$ Note that the bounded inclusion $B_{\varepsilon}^{2, r}\left(\mathbb{R}^{n}\right) \hookrightarrow B_{\varepsilon}^{2, \infty}\left(\mathbb{R}^{n}\right)$ and (24) provide an a priori bound for $\left|A_{j}\right|_{B_{\varepsilon}^{2, \infty}}$ depending on $M$.
} 
On one hand, note that Hölder inequality and (31) imply

$$
\begin{aligned}
\mid \int_{B} i e^{i \varphi} & \left(A_{1}-\left(A_{2}+\nabla \psi\right)\right) \cdot\left(u_{1} \nabla \overline{u_{2}}-\overline{u_{2}} \nabla u_{1}\right) d x \mid \\
\lesssim & \left\|A_{1}-\left(A_{2}+\nabla \psi\right)\right\|_{L^{n}}\left(\left\|e^{\Phi_{1}^{\sharp}}+r_{1}\right\| L_{L^{d}}\left\|\nabla\left(e^{\Phi_{2}^{\sharp}}+r_{2}\right)\right\|_{L^{2}}\right. \\
& +\sum_{j \neq k}\left\|e^{\Phi_{j}^{\sharp}}+r_{j}\right\|_{L^{d}} h^{-1}\left\|e_{k}^{\Phi^{\sharp}}+r_{k}\right\|_{L^{2}} \\
& \left.+\left\|e^{\Phi_{2}^{\sharp}}+r_{2}\right\|_{L^{d}}\left\|\nabla\left(e^{\Phi_{1}^{\sharp}}+r_{1}\right)\right\|_{L^{2}}\right)
\end{aligned}
$$

since $\zeta_{1}+\overline{\zeta_{2}}=h i \xi$. Here $d=2 n /(n-2)$. Then, since $B$ is bounded, estimate (9), Sobolev's embedding and (14) imply that

$$
\begin{gathered}
\left|\int_{B} i e^{i \varphi}\left(A_{1}-\left(A_{2}+\nabla \psi\right)\right) \cdot\left(u_{1} \nabla \overline{u_{2}}-\overline{u_{2}} \nabla u_{1}\right) d x\right| \\
\lesssim\left\|A_{1}-\left(A_{2}+\nabla \psi\right)\right\|_{L^{n}} h^{-(\varepsilon+4) /(\varepsilon+2)} .
\end{gathered}
$$

On the other hand, Hölder inequality and (31) imply again

$$
\begin{aligned}
\mid \int_{B} e^{i \varphi}\left(A_{1}^{2}\right. & \left.-\left(A_{2}+\nabla \psi\right)^{2}\right) u_{1} \overline{u_{2}} d x \mid \\
& \lesssim\left\|A_{1}^{2}-\left(A_{2}+\nabla \psi\right)^{2}\right\|_{L^{n / 2}}\left\|e^{\Phi_{1}^{\sharp}}+r_{1}\right\|_{L^{d}}\left\|e^{\Phi_{2}^{\sharp}}+r_{2}\right\|_{L^{d}}
\end{aligned}
$$

since $\zeta_{1}+\overline{\zeta_{2}}=h i \xi$. Once again, since $B$ is bounded, estimate (9), Sobolev's embedding, (14), (31) and the a priori estimate applied to $\left\|A_{1}+A_{2}+\nabla \psi\right\|_{L^{n}}$ imply that

$$
\begin{aligned}
\mid \int_{B} e^{i \varphi}\left(A_{1}^{2}-\left(A_{2}+\nabla \psi\right)^{2}\right) & u_{1} \overline{u_{2}} d x \mid \\
& \lesssim\left\|A_{1}-\left(A_{2}+\nabla \psi\right)\right\|_{L^{n}} h^{-4 /(\varepsilon+2)} .
\end{aligned}
$$

Because of the same reasons we have

$$
\begin{aligned}
\mid \int_{B} e^{i \varphi}\left(A_{1}-A_{2}-\nabla \psi\right) \cdot \nabla \varphi & u_{1} \overline{u_{2}} d x \mid \\
& \lesssim\left\|A_{1}-\left(A_{2}+\nabla \psi\right)\right\|_{L^{n}} h^{-4 /(\varepsilon+2)} .
\end{aligned}
$$

By elementary interpolation, we know that

$$
\left\|A_{1}-\left(A_{2}+\nabla \psi\right)\right\|_{L^{n}} \leq\left\|A_{1}-\left(A_{2}+\nabla \psi\right)\right\|_{L^{2}}^{\theta}\left\|A_{1}-\left(A_{2}+\nabla \psi\right)\right\|_{L^{p}}^{1-\theta},
$$

where $p$ is chosen to satisfies $1 / n=\theta / 2+(1-\theta) / p$. Note that $p>n$. Now estimates (28) and (29) imply that

$$
\left\|A_{1}-\left(A_{2}+\nabla \psi\right)\right\|_{L^{n}} \lesssim\left\|d\left(A_{1}-A_{2}\right)\right\|_{H^{-1} \boldsymbol{\Omega}^{2}(B)}^{\theta} .
$$


Recall that $\varphi=\chi\left(\psi-\psi^{*}\right)$ and set $\varphi^{\prime}=(1-\chi)\left(\psi-\psi^{*}\right)$. Since $\nabla \psi=\nabla \varphi+\nabla \varphi^{\prime}$ we get, by Lemma 5.1, that

$$
\begin{aligned}
I & \lesssim \operatorname{dist}\left(C_{1}, C_{2}\right)\left\|u_{1}\right\|_{H^{1}(\Omega)}\left\|u_{2}\right\|_{H^{1}(\Omega)} \\
& +\left|\int_{B} i e^{i \varphi} \nabla \varphi^{\prime} \cdot\left(u_{1} \nabla \overline{u_{2}}-\overline{u_{2}} \nabla u_{1}\right) d x+e^{i \varphi}\left(\nabla \varphi+\nabla \varphi^{\prime}\right) \cdot \nabla \varphi^{\prime} u_{1} \overline{u_{2}} d x\right|
\end{aligned}
$$

The same arguments we used to estimate (33), (34) and (35) yield

$$
I \lesssim \operatorname{dist}\left(C_{1}, C_{2}\right)\left\|u_{1}\right\|_{H^{1}(\Omega)}\left\|u_{2}\right\|_{H^{1}(\Omega)}+\left\|\nabla \varphi^{\prime}\right\|_{L^{n}} h^{-(\varepsilon+4) /(\varepsilon+2)}
$$

Note that

$$
\left\|\nabla \varphi^{\prime}\right\|_{L^{n}} \lesssim\left\|\psi-\psi^{*}\right\|_{W^{1, n}\left(B \backslash \overline{B^{\prime}}\right)}
$$

and

$$
\begin{aligned}
\left\|\psi-\psi^{*}\right\|_{W^{1, n}\left(B \backslash \overline{B^{\prime}}\right)} & \lesssim\left\|\psi-\psi^{*}\right\|_{H^{1}\left(B \backslash \overline{B^{\prime}}\right)}^{\theta}\left\|\psi-\psi^{*}\right\|_{W^{1, p}(B)}^{1-\theta} \\
& \lesssim\left\|d\left(A_{1}-A_{2}\right)\right\|_{H^{-1} \Omega^{2}(B)}^{\theta}
\end{aligned}
$$

by Hölder's inequality, (30) and (28).

Thus, (32), (33), (34), (35), (36), (37), (15), (38) and (39) imply

$$
\begin{aligned}
\mid \int_{B} e^{i \varphi}\left(q_{1}\right. & \left.-q_{2}\right) u_{1} \overline{u_{2}} d x \mid \\
& \lesssim \operatorname{dist}\left(C_{1}, C_{2}\right) e^{c / h}+\left\|d\left(A_{1}-A_{2}\right)\right\|_{H^{-1} \boldsymbol{\Omega}^{2}(B)}^{\theta} h^{-(\varepsilon+4) /(\varepsilon+2)} .
\end{aligned}
$$

Note that this $c$ denotes a different constant that the one in the statement. By Proposition 4.2 we have

$$
\begin{aligned}
&\left|\int_{B} e^{i \varphi}\left(q_{1}-q_{2}\right) u_{1} \overline{u_{2}} d x\right| \lesssim \operatorname{dist}\left(C_{1}, C_{2}\right) e^{c / h} \\
&+\left|\log \operatorname{dist}\left(C_{1}, C_{2}\right)\right|^{-\tilde{c} \theta \varepsilon^{2} / n} h^{-(\varepsilon+4) /(\varepsilon+2)} .
\end{aligned}
$$

On the other hand, using (9) and (14) we see that

$$
\left|\int_{B} e^{i \varphi}\left(q_{1}-q_{2}\right) e^{i \xi \cdot x} e^{\Phi_{1}^{\sharp}+\overline{\Phi_{2}^{\sharp}}} d x\right| \lesssim\left|\int_{B} e^{i \varphi}\left(q_{1}-q_{2}\right) u_{1} \overline{u_{2}} d x\right|+h^{\varepsilon /(\varepsilon+2)}
$$

since $\zeta_{1}+\overline{\zeta_{2}}=h i \xi$. Moreover,

$$
\begin{aligned}
\left|\int_{B}\left(q_{1}-q_{2}\right) e^{i \xi \cdot x} d x\right| & \lesssim\left|\int_{B}\left(q_{1}-q_{2}\right) e^{i \xi \cdot x}\left(1-e^{\Phi_{1}+\overline{\Phi_{2}}+i \varphi}\right) d x\right| \\
& +\left|\int_{B} e^{i \varphi}\left(q_{1}-q_{2}\right) e^{i \xi \cdot x}\left(e^{\Phi_{1}+\overline{\Phi_{2}}}-e^{\Phi_{1}^{\sharp}+\overline{\Phi_{2}^{\sharp}}}\right) d x\right| \\
& +\left|\int_{B} e^{i \varphi}\left(q_{1}-q_{2}\right) e^{i \xi \cdot x} e^{\Phi_{1}^{\sharp}+\overline{\Phi_{2}^{\sharp}}} d x\right| .
\end{aligned}
$$

The first term on the right hand side of (42) can be controlled by

$$
\left\|\Phi_{1}+\overline{\Phi_{2}}+i \varphi\right\|_{L^{2}}
$$


using (21), (11) and (31). Furthermore, using (10), we see that

$$
\left(\mu_{1}+i \mu_{2}\right) \cdot \nabla\left(\Phi_{1}+\overline{\Phi_{2}}+i \varphi\right)=i\left(\mu_{1}+i \mu_{2}\right) \cdot\left(A_{2}+\nabla \varphi-A_{1}\right) .
$$

Since $\varphi$ vanishes on $\partial B$, it can be extended by zero out of $B$. Thus, by the boundedness of $\left(\left(\mu_{1}+i \mu_{2}\right) \cdot \nabla\right)^{-1}$ in weighted $L^{2}$ spaces in $\mathbb{R}^{n}$ (see Lemma 3.1 in [22]), we get

$$
\begin{aligned}
\left\|\Phi_{1}+\overline{\Phi_{2}}+i \varphi\right\|_{L^{2}} & \lesssim\left\|A_{2}+\nabla \varphi-A_{1}\right\|_{L^{2}} \\
& \lesssim\left\|A_{2}+\nabla \psi-A_{1}\right\|_{L^{2}}+\left\|\psi-\psi^{*}\right\|_{H^{1}\left(B \backslash \overline{B^{\prime}}\right)} \\
& \lesssim\left|\log \operatorname{dist}\left(C_{1}, C_{2}\right)\right|^{-\tilde{c} \varepsilon^{2} / n} .
\end{aligned}
$$

The last inequality holds because of (29), (30) and Proposition 4.2 ,

The second term on the right hand side of (42) can be estimated by $h^{\varepsilon /(\varepsilon+2)}$ using (21), (12), (9) and (11). Therefore,

$$
\begin{aligned}
\left|\int_{B}\left(q_{1}-q_{2}\right) e^{i \xi \cdot x} d x\right| \lesssim & \left|\log \operatorname{dist}\left(C_{1}, C_{2}\right)\right|^{-\tilde{c} \varepsilon^{2} / n}+h^{\varepsilon /(\varepsilon+2)} \\
& +\left|\int_{B} e^{i \varphi}\left(q_{1}-q_{2}\right) e^{i \xi \cdot x} e^{\Phi_{1}^{\sharp}+\overline{\Phi_{2}^{\sharp}}} d x\right| .
\end{aligned}
$$

Now the result follows directly from (43), (41) and (40).

We next derive the stability estimate for the difference of the electric potentials in the Sobolev space $H^{-\lambda}\left(\mathbb{R}^{n}\right)$ with $\lambda>0$ using the equivalent norm given by

$$
\|u\|_{H^{-\lambda}\left(\mathbb{R}^{n}\right)}^{2}=\int_{\mathbb{R}^{n}}\left(1+|\xi|^{2}\right)^{-\lambda}|\widehat{u}(\xi)|^{2} d \xi
$$

for functions.

Proposition 5.4. Consider $\lambda \in(0,1]$ and $\theta \in(0,2 / n)$. There exist constants $0<\tilde{c}<1$ universal and $c>1$ depending on $\Omega, B, n$ and $\theta$ such that

$$
\left\|q_{1}-q_{2}\right\|_{H^{-\lambda}\left(\mathbb{R}^{n}\right)} \lesssim\left|\log \operatorname{dist}\left(C_{1}, C_{2}\right)\right|^{-\tilde{c} \theta \varepsilon^{3} \lambda / n^{2}},
$$

provided that

$$
\left|\log \operatorname{dist}\left(C_{1}, C_{2}\right)\right|^{-3 \tilde{c} \theta \varepsilon^{2} / n} \leq 1 / c \min \left(1, h_{0}(c M)\right) .
$$

Note that the implicit constant above also depends on $\lambda$ and $\theta$.

Proof. Let $B_{\rho}$ be denote the ball centred at $0 \in \mathbb{R}^{n}$ of radius $\rho \geq 1$ and let $B_{\rho}^{c}$ denote its complement in $\mathbb{R}^{n}$. Let $q$ denote $q_{1}-q_{2}$ for clarity. Using Lemma 5.3 we may estimate

$$
\begin{aligned}
\int_{B_{\rho}} \frac{|\widehat{q}(\xi)|^{2}}{\left(1+|\xi|^{2}\right)^{\lambda}} d \xi \lesssim \rho^{n}( & \operatorname{dist}\left(C_{1}, C_{2}\right) e^{c / h}+h^{\varepsilon /(\varepsilon+2)} \\
& \left.+\left|\log \operatorname{dist}\left(C_{1}, C_{2}\right)\right|^{-\tilde{c} \theta \varepsilon^{2} / n} h^{-5 / 2}\right)^{2}
\end{aligned}
$$


for all $h \leq \min \left(1,2 / \rho, h_{0}(c M)\right)$. Note that $c$ and $\tilde{c}$ here are different to the ones in the statement. On the other hand,

$$
\int_{B_{\rho}^{c}} \frac{|\widehat{q}(\xi)|^{2}}{\left(1+|\xi|^{2}\right)^{\lambda}} d \xi \lesssim \rho^{-2 \lambda}
$$

Choosing

$$
\rho=h^{-\frac{2 \varepsilon}{(2+\varepsilon)(n+2 \lambda)}}
$$

we get

$$
\begin{aligned}
\|q\|_{H^{-\lambda}\left(\mathbb{R}^{n}\right)}^{2} \lesssim & \operatorname{dist}\left(C_{1}, C_{2}\right)^{2} e^{c^{\prime} / h}+h^{4 \varepsilon \lambda /((\varepsilon+2)(2 \lambda+n))} \\
& +\left|\log \operatorname{dist}\left(C_{1}, C_{2}\right)\right|^{-2 \tilde{c} \theta \varepsilon^{2} / n} h^{-6}
\end{aligned}
$$

for $c^{\prime}>2 c$. Note that this choice of $\rho$ satisfies the restriction $\rho \leq 2 / h$. Finally, we set

$$
h=c^{\prime}\left|\log \operatorname{dist}\left(C_{1}, C_{2}\right)\right|^{-\tilde{c} \theta \varepsilon^{2} /(6 n)}
$$

to prove the statement.

We next derive the stability estimate for the difference of the electric potentials in the Besov space $B_{0}^{2, r}\left(\mathbb{R}^{n}\right)$ with $r \in[1,+\infty)$ or $r=\infty$. We use for that the equivalent norm given by

$$
\|u\|_{B_{0}^{2, r}\left(\mathbb{R}^{n}\right)}^{r}=\sum_{j \in \mathbb{N}}\left\|\Delta_{j} u\right\|_{L^{2}\left(\mathbb{R}^{n}\right)}^{r}
$$

for $r \in[1,+\infty)$ and by

$$
\|u\|_{B_{0}^{2, \infty}\left(\mathbb{R}^{n}\right)}=\sup _{j \in \mathbb{N}}\left\|\Delta_{j} u\right\|_{L^{2}\left(\mathbb{R}^{n}\right)}
$$

for $r=\infty$. The family of operators $\left\{\Delta_{j}\right\}_{j \in \mathbb{N}}$ was described right before Proposition 4.3.

In order to ensure the stability for the electric potentials in $B_{0}^{2, r}\left(\mathbb{R}^{n}\right)$, we will assume that $q_{j} \in B_{\varepsilon}^{2, r}\left(\mathbb{R}^{n}\right)$ and

$$
\left\|q_{j}\right\|_{B_{\varepsilon}^{2, r}\left(\mathbb{R}^{n}\right)} \leq M
$$

for $j \in\{1,2\}$.

Proposition 5.5. Let $\theta$ belong to $(0,2 / n)$ and consider $r \in[1,+\infty)$ or $r=\infty$. There exist constants $0<\tilde{c}<1$ universal and $c>1$ depending on $\Omega, B, n$ and $\theta$ such that

$$
\left\|q_{1}-q_{2}\right\|_{B_{0}^{2, r}\left(\mathbb{R}^{n}\right)} \lesssim\left|\log \operatorname{dist}\left(C_{1}, C_{2}\right)\right|^{-\tilde{c} \theta \varepsilon^{4} / n^{2}},
$$

provided that

$$
\left|\log \operatorname{dist}\left(C_{1}, C_{2}\right)\right|^{-36 \tilde{c} \theta \varepsilon^{2} / n} \leq 1 / c \min \left(1, h_{0}(c M)\right)
$$

Note that the implicit constant above also depends on $\theta$. 
Proof. Let $q$ denote $q_{1}-q_{2}$ for clarity. Consider $k \in \mathbb{N}$ to be chosen later. For any $j \in \mathbb{N}$ such that $j \leq k$ we have by Lemma 5.3 that

$$
\begin{aligned}
\left\|\Delta_{j} q\right\|_{L^{2}\left(\mathbb{R}^{n}\right)} \lesssim 2^{k n / 2}( & \operatorname{dist}\left(C_{1}, C_{2}\right) e^{c / h}+h^{\varepsilon /(\varepsilon+2)} \\
& \left.+\left|\log \operatorname{dist}\left(C_{1}, C_{2}\right)\right|^{-\tilde{c} \theta \varepsilon^{2} / n} h^{-5 / 2}\right)
\end{aligned}
$$

for all $h \leq \min \left(2^{-k}, h_{0}(c M)\right)$. Note that $c$ and $\tilde{c}$ do not denote the ones in the statement. On the other hand, if $j>k$, then

$$
\left\|\Delta_{j} q\right\|_{L^{2}\left(\mathbb{R}^{n}\right)} \leq 2^{-k \varepsilon} 2^{j \varepsilon}\left\|\Delta_{j} q\right\|_{L^{2}\left(\mathbb{R}^{n}\right)} .
$$

Thus,

$$
\begin{aligned}
\|q\|_{B_{0}^{2, r}\left(\mathbb{R}^{n}\right)} \lesssim 2^{k n}( & \operatorname{dist}\left(C_{1}, C_{2}\right) e^{c / h}+h^{\varepsilon /(\varepsilon+2)} \\
& \left.+\left|\log \operatorname{dist}\left(C_{1}, C_{2}\right)\right|^{-\tilde{c} \theta \varepsilon^{2} / n} h^{-5 / 2}\right)+2^{-k \varepsilon}
\end{aligned}
$$

for all $h \leq \min \left(2^{-k}, h_{0}(c M)\right)$. Now choosing $k \in \mathbb{N}$ such that

$$
2^{-(k+1)}<h^{\frac{\varepsilon}{(\varepsilon+2)(\varepsilon+n)}} \leq 2^{-k}
$$

we know that there exists a constant $c^{\prime}>0$ such that

$$
\begin{aligned}
\|q\|_{B_{0}^{2, r}\left(\mathbb{R}^{n}\right)} \lesssim & \operatorname{dist}\left(C_{1}, C_{2}\right) e^{c^{\prime} / h}+h^{\varepsilon^{2} /((\varepsilon+2)(\varepsilon+n))} \\
& +\left|\log \operatorname{dist}\left(C_{1}, C_{2}\right)\right|^{-\tilde{c} \theta \varepsilon^{2} / n} h^{-3} .
\end{aligned}
$$

Note that the choice of $k$ satisfies the restriction $h \leq 2^{-k}$. Finally, we set

$$
h=2 c^{\prime}\left|\log \operatorname{dist}\left(C_{1}, C_{2}\right)\right|^{-\tilde{c} \theta \varepsilon^{2} /(6 n)}
$$

to prove the statement.

\section{Estimating the CO-EXACT PART OF the Magnetic POTENTIAL}

This section is devoted to proving Proposition [5.2, by giving the Hodge type decomposition $A_{1}-A_{2}=\delta F+\nabla \psi$. The proof will be split in to two lemmas. The first lemma gives the above decomposition and the rough idea is to choose the exact part $\nabla \psi$ in such a way that it is the sum of the exact component of a Hodge decomposition of $A_{1}-A_{2}$ which vanishes on $\partial B$ and the exact expression of its harmonic component. The other lemma is then devoted to showing that we can estimate the norm of the co-exact part $\delta F$, by the norm of $d A_{1}-d A_{2}$.

We want to point out that the decomposition given in the first lemma, Lemma 6.1, is slightly different from the usual Hodge-MorreyFriedrichs decomposition in bounded domains with smooth boundaries (see for example [19]). This decomposition usually has a harmonic component whose norm might be difficult to estimate. However, in our case we are dealing with a domain with a straightforward topology, i.e. a ball, and the harmonic part can be written as an exact form and its norm can be controlled. 
Another consequence of the simple topology we are dealing with, is that the spaces

$$
\begin{aligned}
& \mathscr{H}_{D}^{1}(B)=\left\{v \in H^{1} \Omega^{1}(B): d v=0, \delta v=0, \mathbf{t} v=0\right\} \\
& \mathscr{H}_{N}^{1}(B)=\left\{v \in H^{1} \Omega^{1}(B): d v=0, \delta v=0, \mathbf{n} v=0\right\}
\end{aligned}
$$

are just the trivial ones, that is, $\mathscr{H}_{D}^{1}(B)=\mathscr{H}_{N}^{1}(B)=\{0\}$. Here $\mathbf{t} v$ and $\mathbf{n} v$ denote the tangential and normal component: 7 of $v$ on $\partial B$. Indeed, if we take $v$ either in $\mathscr{H}_{D}^{1}(B)$ or in $\mathscr{H}_{N}^{1}(B)$, we know, by Theorem 2.2.6 (c) or by Theorem 2.2.7 (a) in [19], that $v$ is smooth. By Poincaré's lemma for closed smooth forms on contractible domains in $\mathbb{R}^{n}$, we have that $v=d g$ with $g$ a smooth function. In consequence, we have that $-\Delta g=\delta d g=0$ satisfying either $\mathbf{t} d g=0$ or $\mathbf{n} d g=0$, which implies that $g$ is constant in $B$. Thus $v=0$. The fact that $\mathscr{H}_{D}^{1}(B)=$ $\mathscr{H}_{N}^{1}(B)=\{0\}$ will be relevant when referring to the results in [19] since some arguments will become simpler (it is here where the topology of $B$ is playing its role).

Lemma 6.1. Let $A_{1}, A_{2} \in L^{\infty} \Omega^{1}(B)$ denote the 1-forms representing the magnetic potentials. Then there exist $\psi \in W^{1, p}(B)$ and $F \in$ $W^{1, p} \Omega^{2}(B)$ such that

$$
A_{1}-A_{2}=d \psi+\delta F
$$

$\mathbf{n} F=0$ and

$$
\|\psi\|_{W^{1, p}(B)} \lesssim\left\|A_{1}-A_{2}\right\|_{L^{p} \boldsymbol{\Omega}^{1}(B)}
$$

for all $p \geq 2$. Here $\mathbf{n} F$ denote the normal component of $F$ on $\partial B$ and $\delta$ the co-differential.

Moreover, if there exists a ball $B^{\prime}$ containing supp $A_{j}$ with $j \in\{1,2\}$ and such that $\overline{B^{\prime}} \subset B$, then

$$
\left\|\psi-\psi^{*}\right\|_{W^{1, p}\left(B \backslash \overline{B^{\prime}}\right)} \lesssim\|\delta F\|_{L^{p} \boldsymbol{\Omega}^{1}(B)},
$$

where $\psi^{*}$ denotes the average of $\psi$ in $B \backslash \overline{B^{\prime}}$.

Proof. Through out the proof we will follow most of the notation use in [19] and we will refer to it several times.

Let $u$ belong to $L^{p} \Omega^{1}(B)$ with $p \geq 2$, we want to write $u=d g+\delta f+$ $d h$ with $\mathbf{t} g=0, \mathbf{n} f=0$ and $d h$ harmonic. Since $u$ belongs to $L^{2} \Omega^{1}(B)$ and $\mathscr{H}_{D}^{1}(B)=\mathscr{H}_{N}^{1}(B)=\{0\}$, we have by Theorem 2.2.4 and Theorem 2.2.7 (b) in [19] that:

(a) There exists a unique $\phi_{D} \in H^{1} \Omega_{D}^{1}(B)$ (the space of forms in $H^{1} \Omega^{1}(B)$ with vanishing tangential components on $\partial B$ ) such that

$$
\int_{B}\left\langle d \phi_{D}, d v\right\rangle+\delta \phi_{D} \delta v d x=\int_{B}\langle u, v\rangle d x
$$

\footnotetext{
${ }^{7}$ More details about tangential and normal components can be found in [19].
} 
for any $v \in H^{1} \Omega_{D}^{1}(B)$. The solution $\phi_{D}$ is usually called the Dirichlet potential of $u$. Note that $u$ is uniquely determined by its Dirichlet potential.

(b) There exists a unique $\phi_{N} \in H^{1} \Omega_{N}^{1}(B)$ (the space of forms in $H^{1} \Omega^{1}(B)$ with vanishing normal components on $\partial B$ ) such that

$$
\int_{B}\left\langle d \phi_{N}, d v\right\rangle+\delta \phi_{N} \delta v d x=\int_{B}\langle u, v\rangle d x
$$

for any $v \in H^{1} \Omega_{N}^{1}(B)$. The solution $\phi_{N}$ is usually called the Neumann potential of $u$. Note that $u$ is uniquely determined by its Neumann potential.

By Theorem 2.2.5 (a) and Theorem 2.2.7 (b) in [19, we know that $\phi_{D}$ and $\phi_{N}$ belong to $H^{2} \Omega^{1}(B)$. Moreover, integrating by parts in (a) and (b) above, we see that $\mathbf{t} \delta \phi_{D}=0$ and $\mathbf{n} d \phi_{N}=0$. Finally, by Theorem 2.2.6 (a) and Theorem 2.2.7 (c) in [19], we know that $\phi_{D}$ and $\phi_{N}$ belong to $W^{2, p} \Omega^{1}(B)$ for $p \geq 2$.

Define $g=\delta \phi_{D}, f=d \phi_{N}$ and $w=u-d g-\delta f$, then $\mathbf{t} g=0, \mathbf{n} f=0$ and $w \in\left(\mathscr{E}^{1}(B) \oplus \mathscr{C}^{1}(B)\right)^{\perp}$ (orthogonality in the sense of $L^{2} \Omega^{1}(B)$ ) with

$$
\mathscr{E}^{1}(B)=\left\{d v: v \in H_{0}^{1}(B)\right\}, \quad \mathscr{C}^{1}(B)=\left\{\delta v: v \in H^{1} \Omega_{N}^{2}(B)\right\}
$$

and $H^{1} \Omega_{N}^{2}(B)$ denoting the space of forms in $H^{1} \Omega^{2}(B)$ with vanishing normal components on $\partial B$. The fact that $w$ is in the orthogonal complement of $\mathscr{E}^{1}(B) \oplus \mathscr{C}^{1}(B)$ is proven in the proof of Lemma 2.4.3 (a) in [19]. Therefore, we have that $d g \in \mathscr{E}^{1}(B) \cap L^{p} \Omega^{1}(B), \delta f \in$ $\mathscr{C}^{1}(B) \cap L^{p} \Omega^{1}(B)$ and $w \in\left(\mathscr{E}^{1}(B) \oplus \mathscr{C}^{1}(B)\right)^{\perp} \cap L^{p} \boldsymbol{\Omega}^{1}(B)$. Furthermore, Theorem 2.4.5 (a) in [19] states that $\left(\mathscr{E}^{1}(B) \oplus \mathscr{C}^{1}(B)\right)^{\perp}=L^{2} \mathscr{H}^{1}(B)$, where $L^{2} \mathscr{H}^{1}(B)$ is defined as the closure of

$$
\mathscr{H}^{1}(B)=\left\{v \in H^{1} \Omega^{1}(B): d v=0, \delta v=0\right\}
$$

in $L^{2} \Omega^{1}(B)$.

We next show that $w=d h$ with $h \in W^{1, p}(B)$ (note that this will be possible because of the topology of $B$ ). Indeed, let $\phi$ denote the Neumann potential of $w$. By the arguments given above, we know that $\phi \in W^{2, p} \boldsymbol{\Omega}^{1}(B) \cap H^{1} \Omega_{N}^{1}(B)$ and $\mathbf{n} d \phi=0$. Defining $h=\delta \phi$ and noting that $w=\delta d \phi+d \delta \phi$, we immediately see that $w-d h=\delta d \phi$. Note that $d \phi \in H^{1} \Omega_{N}^{2}(B)$ and satisfies

$$
\int_{B}\langle d(d \phi), d v\rangle+\langle\delta(d \phi), \delta v\rangle d x=\int_{B}\langle w-d h, \delta v\rangle d x=0
$$

for all $v \in H^{1} \Omega_{N}^{2}(B)$-last identity follows from a density argument together with the Green formula stated in Proposition 2.1.2 in [19]. This means that $d \phi$ is a weak solution of the Hodge-Laplacian with zero Neumann boundary condition and zero right hand side. By Theorem 
2.2.7 (b) in [19], we know that the unique solution for this problem in $\mathscr{H}_{N}^{2}(B)^{\perp} \cap H^{1} \Omega_{N}^{2}(B)$ is the trivial one. Thus, if $d \phi \in \mathscr{H}_{N}^{2}(B)^{\perp}$ with

$$
\mathscr{H}_{N}^{2}(B)=\left\{v \in H^{1} \Omega^{2}(B): d v=0, \delta v=0, \mathbf{n} v=0\right\},
$$

then $d \phi=0$ and consequently $w=d h$. Finally, the fact that $d \phi$ belongs to $\mathscr{H}_{N}^{2}(B)^{\perp}$ is a simple consequence of the Green formula stated in Proposition 2.1.2 in [19].

By now, we know that $u \in L^{p} \Omega^{1}(B)$ with $p \geq 2$ can be written as

$$
u=d g+\delta f+d h
$$

with $d g \in \mathscr{E}^{1}(B) \cap L^{p} \Omega^{1}(B), \delta f \in \mathscr{C}^{1}(B) \cap L^{p} \Omega^{1}(B)$ and $d h \in$ $L^{2} \mathscr{H}^{1}(B) \cap L^{p} \boldsymbol{\Omega}^{1}(B)$. We next want to estimate $g, f$ and $h$ in terms of $u$. This will be achieved using a simple consequence of the open mapping theorem that can be stated as follows. Let $X$ and $Y$ be two Banach spaces and let $T: X \longrightarrow Y$ be a bounded linear operator. If $T$ is bijective, then the inverse of $T$ is bounded. Let $X_{D}$ and $X_{N}$ denote the spaces

$$
\begin{aligned}
& X_{D}=\left\{v \in W^{2, p} \Omega^{1}(B) \cap H^{1} \Omega_{D}^{1}(B): \mathbf{t} \delta v=0\right\}, \\
& X_{N}=\left\{v \in W^{2, p} \Omega^{1}(B) \cap H^{1} \Omega_{N}^{1}(B): \mathbf{n} d v=0\right\} ;
\end{aligned}
$$

which endowed with the norm of $W^{2, p} \Omega^{1}(B)$ become Banach spaces. On the other hand, consider $Y_{D}=Y_{N}=L^{p} \Omega^{1}(B)$. Defining the operators $T_{D}$ and $T_{N}$ as

$$
T_{D} v=-\Delta v \quad \forall v \in X_{D}, \quad T_{N} v=-\Delta v \quad \forall v \in X_{N}
$$

we see that they are bounded and linear. Moreover, by the discussion given above about the existence, uniqueness and regularity of the Dirichlet and Neumann potentials respectively, we know that $T_{D}$ and $T_{N}$ are bijective whenever $p \geq 2$. Therefore, since $g=\delta \phi_{D}, f=d \phi_{N}$ and $h=\delta \phi$ with $\phi_{D}$ and $\phi_{N}$ the Dirichlet and Neumann potentials for $u$ and $\phi$ the Neumann potential for $w$, we have that

$$
\|g\|_{W^{1, p}(B)}+\|f\|_{W^{1, p} \boldsymbol{\Omega}^{2}(B)}+\|h\|_{W^{1, p}(B)} \lesssim\|u\|_{L^{p} \boldsymbol{\Omega}^{1}(B)} .
$$

Now since $A_{1}-A_{2} \in L^{\infty} \Omega^{1}(B)$ the above argument can be performed for all $p \geq 2$ which provides a proof for the first part of the statement.

Finally, the second part of the statement is a simple consequence of Poincaré's inequality (see [10]) and the fact that $\left.d \psi\right|_{B \backslash \overline{B^{\prime}}}=\left.\delta F\right|_{B \backslash \overline{B^{\prime}}}$ (since $A_{j}$ is zero outside $\Omega$ ).

We now use the properties summarized in Lemma 6.1 and its proof to derive an estimate for the co-exact part of the decomposition (44). One of the key elements of the proof is the Friedrich type inequality labelled as (52) below. 
Lemma 6.2. Let $A_{1}, A_{2}$ and $\psi$ be as in Lemma 6.1 (including the conditions for $\operatorname{supp} A_{j}$ ). Then the following estimate holds

$$
\left\|A_{1}-\left(A_{2}+d \psi\right)\right\|_{L^{2} \boldsymbol{\Omega}^{1}(B)} \lesssim\left\|d\left(A_{1}-A_{2}\right)\right\|_{H^{-1} \boldsymbol{\Omega}^{2}(B)} \cdot
$$

Moreover, if $B^{\prime}$ and $\psi^{*}$ are as in Lemma 6.1, we have that

$$
\left\|\psi-\psi^{*}\right\|_{H^{1}\left(B \backslash \overline{B^{\prime}}\right)} \lesssim\left\|d\left(A_{1}-A_{2}\right)\right\|_{H^{-1} \Omega^{2}(B)} .
$$

Proof. Note that the second part of the statement is an immediate consequence of Lemma 6.1 and (48).

The idea to prove the first part is roughly speaking the following: $d\left(A_{1}-A_{2}\right)=d \delta F=-\Delta F$ since $F=d \phi_{N}$, where $\phi_{N}$ is the Neumann potential of $A_{1}-A_{2}$. Then, one should be able control $\|\delta F\|_{L^{2} \Omega^{1}(B)}$ by $\left\|d\left(A_{1}-A_{2}\right)\right\|_{H^{-1} \Omega^{2}(B)}$ since $A_{1}-A_{2}$ has compact support inside $B^{\prime}$. Let us now give a rigorous proof.

Consider a sequence $\left\{u_{m}\right\} \subset C_{0}^{\infty} \Omega^{1}(B)$ converging to $A_{1}-A_{2}$ in $L^{2} \Omega^{1}(B)$ such that $\operatorname{supp} u_{m} \subset B^{\prime}$ for all $m \in \mathbb{N}$. This is possible because $A_{1}-A_{2}$ vanishes out of $\Omega$. Let $u_{m}$ be decomposed as in the proof of Lemma 6.1, that is, $u_{m}=d\left(g_{m}+h_{m}\right)+\delta f_{m}$. Since the decomposition is orthogonal in $L^{2} \Omega^{1}(B)$ (see Theorem 2.4.2 in [19]), $\delta f_{m}$ converges to $\delta F$ in $L^{2} \Omega^{1}(B)$ as $m$ goes to infinity. Since $f_{m}=d \phi_{N}^{m}$ with $\phi_{N}^{m}$ denoting the Neumann potential of $u_{m}$, we have that $f_{m} \in$ $\mathscr{H}_{N}^{2}(B)^{\perp}$ and

$$
\begin{aligned}
\int_{B}\left\langle d f_{m}, d v\right\rangle+\left\langle\delta f_{m}, \delta v\right\rangle d x & =\int_{B}\left\langle u_{m}-d\left(g_{m}+h_{m}\right), \delta v\right\rangle d x \\
& =\int_{B}\left\langle d u_{m}, v\right\rangle d x
\end{aligned}
$$

for all $v \in H^{1} \Omega_{N}^{2}(B)$-both facts are consequence of the Green formula in Proposition 2.1.2 in [19]. Consider now a cut-off function $\chi \in C_{0}^{\infty}(B)$ such that $\chi(x)=1$ for all $x \in B^{\prime}$. Then,

$$
\begin{aligned}
\left|\int_{B}\left\langle d u_{m}, v\right\rangle d x\right| & =\left|\int_{B}\left\langle d u_{m}, \chi v\right\rangle d x\right| \\
& \lesssim\left\|d u_{m}\right\|_{H^{-1} \boldsymbol{\Omega}^{2}(B)}\|v\|_{H^{1} \boldsymbol{\Omega}^{2}(B)}
\end{aligned}
$$

for all $v \in H^{1} \Omega_{N}^{2}(B)$. On the other hand, by Proposition 2.2.3 in [19] and the fact that the Hodge star operator, denoted by $*$, is an $H^{1}$-isometry and satisfies $* \mathbf{t}=\mathbf{n} *$, we know that

$$
\left\|f_{m}\right\|_{H^{1} \mathbf{\Omega}^{2}(B)}^{2} \lesssim \int_{B}\left\langle d f_{m}, d f_{m}\right\rangle+\left\langle\delta f_{m}, \delta f_{m}\right\rangle d x
$$

since $f_{m} \in \mathscr{H}_{N}^{2}(B)^{\perp} \cap H^{1} \Omega_{N}^{2}(B)$. Now by (152), (501), (151) and the continuity of $d$ as operator from $L^{2} \Omega^{1}(B)$ to $H^{-1} \Omega^{2}(B)$, we get

$$
\left\|f_{m}\right\|_{H^{1} \boldsymbol{\Omega}^{2}(B)} \lesssim\left\|d\left(A_{1}-A_{2}\right)\right\|_{H^{-1} \boldsymbol{\Omega}^{2}(B)}+\left\|u_{m}-\left(A_{1}-A_{2}\right)\right\|_{L^{2} \boldsymbol{\Omega}^{1}(B)} .
$$


Since the second term on the right hand side of last estimate tends to vanish as $m$ grows, the sequence $\left\{f_{m}\right\}$ is bounded. By a standard compactness argument, there exist a subsequence $\left\{f_{m_{k}}\right\}$ and $f \in H^{1} \Omega^{2}(B)$ such that $f_{m_{k}}$ converges to $f$ in $L^{2} \Omega^{2}(B)$ as $k$ goes to infinity and

$$
\|f\|_{H^{1} \boldsymbol{\Omega}^{2}(B)} \lesssim\left\|d\left(A_{1}-A_{2}\right)\right\|_{H^{-1} \boldsymbol{\Omega}^{2}(B)} .
$$

Finally, by the continuity of $\delta$ as operator from $L^{2} \Omega^{2}(B)$ to $H^{-1} \Omega^{1}(B)$ and the uniqueness of the limit in the latter space we have that $\delta f=$ $\delta F$. Thus, the first part of the lemma follows from (53) and Lemma 6.1 .

Remark 6.3. Note that the condition of $A_{1}-A_{2}$ having support in $\bar{\Omega} \subset B^{\prime}$ is fundamental to obtain (48). Otherwise, estimate (51) would become

$$
\left|\int_{B}\left\langle d u_{m}, v\right\rangle d x\right| \lesssim\left\|d u_{m}\right\|_{H^{1} \Omega_{N}^{2}(B)^{*}}\|v\|_{H^{1} \Omega_{N}^{2}(B)}
$$

with $H^{1} \Omega_{N}^{2}(B)^{*}$ denoting the dual space of $H^{1} \Omega_{N}^{2}(B)$.

Acknowledgments. The authors are supported by the projects ERC2010 Advanced Grant, 267700 - InvProb and Academy of Finland (Decision number 250215, the Centre of Excellence in Inverse Problems). PC also belongs to the project MTM 2011-02568 Ministerio de Ciencia y Tecnología de España.

\section{REFERENCES}

[1] G. Alessandrini, Stable determination of conductivity by boundary measurements, Appl. Anal. 27 (1988), 153-172.

[2] K. Astala and L. Päivärinta, Calderón's inverse conductivity problem in the plane. Ann. of Math. 163 (2006), 265-299.

[3] R. M. Brown and R. H. Torres, Uniqueness in the inverse conductivity problem for conductivities with $3 / 2$ derivatives in $L^{p}, p>2 n$. J. Fourier Anal. Appl. 9 (2003), 563-574.

[4] P. Caro, Stable determination of the electromagnetic coefficients by boundary measurements. Inverse Problems 26 (2010), 105014, 25 pp.

[5] P. Caro, On an inverse problem in electromagnetism with local data: stability and uniqueness. Inverse Probl. Imaging 5 (2011), 297-322.

[6] P. Caro, A. García and J. M. Reyes, Stability of the Calderón problem for less regular conductivities, J. Differential Equations 254 (2013), 469-492.

[7] P. Caro and T. Zhou, On global uniqueness for an IBVP for the time-harmonic Maxwell equations, to appear in Analysis \& PDE.

[8] A. Clop, D. Faraco and A. Ruiz, Stability of Calderón's inverse conductivity problem in the plane for discontinuous conductivities. Inverse Probl. Imaging 4 (2010), 49-91.

[9] G. Eskin and J. Ralston, Inverse scattering problem for the Schrdinger equation with magentic potential at a fixed energy, Commun. Math. Phys. 173 (1995), 100-224.

[10] L. C. Evans, Partial differential equations. American Mathematical Society 1998. 
[11] D. Faraco and K. Rogers, The Sobolev norm of characteristic functions with applications to the Calderón Inverse Problem Q. J. Math., 64 (2013), 133-147.

[12] A. García and G. Zhang, Reconstruction from boundary measurements for less regular conductivities. arXiv:1212.0727.

[13] B. Haberman, D. Tataru, Uniqueness in Calderon's problem with Lipschitz conductivities. Duke Math. J., 162 (2013), 497-516.

[14] V. Isakov, S. Nagayasu, G. Uhlmann and J.-N. Wang, Increasing stability of the inverse boundary value problem for the Schrödinger equation. arXiv:1302.0940 (2013).

[15] K. Krupchyk and G. Uhlmann, Uniqueness in an inverse boundary problem for a magnetic Schrödinger operator with a bounded magnetic potential, to appear in Commun. Math. Phys..

[16] G. Nakamura, Z. Sun and G. Uhlmann, Global identifiability for an inverse problem for the Schrödinger equation in a magnetic field, Math. Ann. 303 (1995), no. 3, 377-388.

[17] A. Panchenko, An inverse problem for the magnetic Schrödinger equation and quasi-exponential solutions of nonsmooth partial differential equations, Inverse Problems 18 (2002), no. 5, 1421-1434.

[18] M. Salo, Inverse problems for nonsmooth first order perturbations of the Laplacian, Ann. Acad. Sci. Fenn. Math. Diss. 139 (2004).

[19] G. Schwarz, Hodge decomposition - A method for solving boundary value problems, Springer 1995.

[20] E. M. Stein, Singular integrals and differentiability properties of functions, Princeton University Press, New Jersey (1970).

[21] Z. Sun, An inverse boundary value problem for Schrödinger operators with vector potentials, Trans. Amer. Math. Soc. 338 (1993), 953-969.

[22] J. Sylvester and G. Uhlmann, A global uniqueness theorem for an inverse boundary value problem, Ann. of Math. 125 (1987), 153-169.

[23] C. Tolmasky, Exponentially growing solutions for nonsmooth first-order perturbations of the Laplacian, SIAM J. Math. Anal. 29 (1998), no. 1, 116-133.

[24] H. Triebel, Function spaces in Lipschitz domains and on Lipschitz manifolds. Characteristic functions as pointwise multipliers, Rev. Mat. Complut. 15 (2002), 475-524.

[25] L. Tzou, Stability estimates for coefficients of the magnetic Schrödinger equation from full and partial boundary measurements, Comm. Partial Differential Equations 33 (2008), 1911-1952.

Department of Mathematics and Statistics, Helsingin Yliopisto / HelsingFors universite / University of Helsinki, Finland

E-mail address: pedro.caro@helsinki.fi

E-mail address: valter.pohjola@helsinki.fi 